\title{
EVOLUTION OF THE SIZES OF GALAXIES OVER $7<z<12$ REVEALED BY THE 2012 HUBBLE ULTRA DEEP FIELD CAMPAIGN
}

\author{
Yoshiaki Ono ${ }^{1}$, Masami Ouchi ${ }^{1,2}$, Emma Curtis-Lake ${ }^{3}$, Matthew A. SchenKer ${ }^{4}$, Richard S. Ellis ${ }^{4}$, \\ Ross J. McLure ${ }^{3}$, James S. Dunlop ${ }^{3}$, Brant E. Robertson ${ }^{5}$, Anton M. Koekemoer ${ }^{6}$, Rebecca A. A. Bowler ${ }^{3}$, \\ Alexander B. Rogers ${ }^{3}$, Evan Schneider ${ }^{5}$, Stephane Charlot $^{7}$, Daniel P. Stark ${ }^{5}$, \\ Kazuhiro Shimasaku ${ }^{8}$, Steven R. Furlanetto ${ }^{9}$, and Michele Cirasuolo ${ }^{3,10}$ \\ ${ }^{1}$ Institute for Cosmic Ray Research, The University of Tokyo, Kashiwa 277-8582, Japan; ono@ icrr.u-tokyo.ac.jp \\ ${ }^{2}$ Kavli Institute for the Physics and Mathematics of the Universe (Kavli IPMU), WPI, The University of Tokyo, Chiba 277-8583, Japan \\ ${ }^{3}$ Institute for Astronomy, University of Edinburgh, Royal Observatory, Edinburgh EH9 3HJ, UK \\ ${ }^{4}$ Department of Astrophysics, California Institute of Technology, MS 249-17, Pasadena, CA 91125, USA \\ ${ }^{5}$ Department of Astronomy and Steward Observatory, University of Arizona, Tucson, AZ 85721, USA \\ ${ }^{6}$ Space Telescope Science Institute, Baltimore, MD 21218, USA \\ ${ }^{7}$ UPMC-CNRS, UMR7095, Institut d'Astrophysique, F-75014 Paris, France \\ ${ }^{8}$ Department of Astronomy, Graduate School of Science, The University of Tokyo, Tokyo 113-0033, Japan \\ ${ }^{9}$ Department of Physics \& Astronomy, University of California, Los Angeles, CA 90095, USA \\ ${ }^{10}$ UK Astronomy Technology Centre, Royal Observatory, Edinburgh EH9 3HJ, UK \\ Received 2012 December 13; accepted 2013 September 27; published 2013 October 24
}

\begin{abstract}
We analyze the redshift- and luminosity-dependent sizes of dropout galaxy candidates in the redshift range $z \sim 7-12$ using deep images from the 2012 Hubble Ultra Deep Field (UDF12) campaign, which offers two advantages over that used in earlier work. First, we utilize the increased signal-to-noise ratio offered by the UDF12 imaging to provide improved measurements for known galaxies at $z \simeq 6.5-8$ in the HUDF. Second, because the UDF12 data have allowed the construction of the first robust galaxy sample in the HUDF at $z>8$, we have been able to extend the measurement of average galaxy size out to higher redshifts. Restricting our measurements to sources detected at $>15 \sigma$, we confirm earlier indications that the average half-light radii of $z \sim 7-12$ galaxies are extremely small, $0.3-0.4 \mathrm{kpc}$, comparable to the sizes of giant molecular associations in local star-forming galaxies. We also confirm that there is a clear trend of decreasing half-light radius with increasing redshift, and provide the first evidence that this trend continues beyond $z \simeq 8$. Modeling the evolution of the average half-light radius as a power law, $\propto(1+z)^{s}$, we obtain a best-fit index of $s=-1.30_{-0.14}^{+0.12}$ over $z \sim 4-12$. A clear size-luminosity relation is evident in our dropout samples. This relation can be interpreted in terms of a constant surface density of star formation over a range in luminosity of $0.05-1.0 L_{z=3}^{*}$. The average star formation surface density in dropout galaxies is $2-3$ orders of magnitude lower than that found in extreme starburst galaxies, but is comparable to that seen today in the centers of normal disk galaxies.
\end{abstract}

Key words: galaxies: evolution - galaxies: formation - galaxies: high-redshift - galaxies: structure

Online-only material: color figures

\section{INTRODUCTION}

Considerable progress has been made in charting the abundance of galaxies at $z \sim 7-10$ from deep imaging with ground-based observations and various campaigns undertaken with the Wide Field Camera 3 infrared channel (WFC3/IR) on Hubble Space Telescope (HST). Sample selection makes use of the well-established dropout technique, which takes advantage of the unique spectral characteristics of high-redshift star-forming galaxies, i.e., a blue UV spectrum and a sharp drop in flux at wavelengths shorter than Ly $\alpha$. These complementary studies have identified a large number of dropout galaxies at $z \sim 7$ and beyond. Investigating the abundance of dropout galaxies over $7<z<10$ has revealed a clear decrease in the number density of luminous galaxies with increasing redshift (e.g., Ouchi et al. 2009; McLure et al. 2010; Castellano et al. 2010; Oesch et al. 2010b; Bouwens et al. 2011b).

Characterizing the evolution of galaxy morphologies and sizes is useful for understanding galaxy formation history. Analytical studies have calculated the size-redshift relation of disk galaxies, suggesting the typical size of galaxies of a given luminosity is expected to decrease with increasing redshift (Mo et al. 1998, 1999). The virial radius of a dark matter halo scales with redshift and virial velocity or virial mass. Assuming that the exponential scale length of the baryonic disk scales with the virial radius, the sizes of disks are expected to scale with redshift, proportional to $H(z)^{-2 / 3}$ at a fixed mass or $H(z)^{-1}$ at a fixed circular velocity (e.g., Ferguson et al. 2004), where $H(z)$ is the Hubble parameter which scales as $\sim(1+z)^{3 / 2}$ at high redshifts.

Earlier observations have reported that the sizes (half-light radii) of dropout galaxies decrease according to about $(1+z)^{-1}$ up to $z \sim 7$ (Oesch et al. 2010a), which is expected at fixed halo masses, and in good agreement with previous estimates at lower redshifts (Bouwens et al. 2004). However, because they used only the first epoch of their survey data with the WFC3/IR, their analysis still shows large uncertainties, especially in their fainter sample. Therefore, it is also consistent with $(1+z)^{-1.5}$ (Ferguson et al. 2004; Hathi et al. 2008), as expected for sizes that scale with halo circular velocity.

Oesch et al. (2010a) have also reported that the star formation rate (SFR) surface densities of dropout galaxies remains constant from $z \sim 7$ to $z \sim 4$. They suggest a possible explanation is that the average star formation efficiency is very similar in all these galaxies, and that feedback effects change the mode of star formation by only a small amount. It would be interesting 
to see if this possible trend continues toward higher redshifts, to infer star formation activities in galaxies at earlier epochs of galaxy formation.

Recently, a new campaign was carried out with the WFC3/IR to significantly deepen the Hubble Ultra Deep Field in 2012 (GO 12498; PI: R. Ellis; hereafter UDF12; see Ellis et al. 2013 and Koekemoer et al. 2013 for the project description); this yields the deepest near-infrared images ever obtained. Additional scientific results from this project are presented in Dunlop et al. (2013), Schenker et al. (2013), McLure et al. (2013), and Robertson et al. (2013). In this paper, we study morphologies of $z \sim 7-12$ galaxies based on the complete WFC3/IR UDF12 data set. The advantages of the new images are (1) a new F140W image and deeper F160W data from which we obtain robust estimates on the rest-frame UV morphologies of galaxies not only at $z \sim 7$, but also $z \sim 8-12$ for the first time, and (2) a deeper F105W image which enables us to safely exclude contaminations by foreground sources from our galaxy samples at $z \sim 8$ and beyond. ${ }^{11}$ The purpose of this paper is to investigate the galaxy size and SFR surface density evolution beyond $z \sim 7$, and the correlation of size with UV luminosity.

The outline of this paper is as follows. After describing the imaging data used in this study in Section 2, we summarize our dropout galaxy samples in Section 3. Our size analysis is described in Section 4. In Section 5, we investigate the size-luminosity relation and the size evolution and discuss the implications. A summary is given in Section 6. Throughout this paper, we use magnitudes in the AB system (Oke \& Gunn 1983) and assume a flat universe with $\Omega_{\mathrm{m}}=0.3, \Omega_{\Lambda}=0.7$, and $H_{0}=70 \mathrm{~km} \mathrm{~s}^{-1} \mathrm{Mpc}^{-1}$. In this cosmological model, an angular dimension of 1.0 arcsec corresponds to a physical dimension of $5.365 \mathrm{kpc}$ at $z=6.7,4.818 \mathrm{kpc}$ at $z=8.0,4.465 \mathrm{kpc}$ at $z=9.0$, and $3.683 \mathrm{kpc}$ at $z=11.9$. We express galaxy UV luminosities in units of the characteristic luminosity of $z \sim 3$ galaxies, $L_{z=3}^{*}$, which corresponds to $M_{1600}=-21.0$ (Steidel et al. 1999). The four WFC3/IR filters we use, F105W, F125W, F140W, and F160W, are denoted by $Y_{105}, J_{125}, J_{140}$, and $H_{160}$, respectively. We also use four Advanced Camera for Surveys (ACS) filters, F435W, F606W, F775W, and F850LP, which are denoted by $B_{435}, V_{606}, i_{775}$, and $z_{850}$, respectively.

\section{OBSERVATIONS}

The primary data set used in this size analysis for $z \sim 7-12$ galaxies is the ultra-deep WFC3/IR observations taken for the UDF12 campaign combined with images taken for the UDF09 campaign (GO 11563; PI: G. Illingworth). In the UDF09 campaign, WFC3/IR data were obtained over three fields: the HUDF main, and two parallel fields. The UDF12 campaign has obtained 128 orbits of WFC3/IR data over the HUDF main field. We have combined all the exposures including the data from other HST programs (GO12060, 12061, 12062; PI: S. Faber, H. Ferguson; GO12099; PI: A. Riess). In total, the observations over the HUDF main field include 253 orbits (F105W: 100 orbits; F125W: 39 orbits; F140W: 30 orbits; F160W: 84 orbits). A more detailed description of the UDF12 data set is provided by Koekemoer et al. (2013), and the final reduced data are being made publicly available as High-Level Science Products ${ }^{12}$ that are delivered to the Space Telescope Science Institute archive,

\footnotetext{
11 We do not use our deep F105W image for the morphology analysis of $z \sim 7$ galaxies, since a redshifted Ly $\alpha$ and the continuum break of an object at $z \gtrsim 6.4$ enters the F105W band.

${ }_{12}$ http://archive.stsci.edu/prepds/hudf $12 /$
}

and further details and current updates about the survey are provided at the project Web site. ${ }^{13}$

To minimize the effects of morphological $K$-correction and take the advantage of the UDF12 campaign, we measure sizes of galaxies in the images of the WFC3/IR band that is the closest to the rest-frame 1600-1700 $\AA$. A stack of the pointspread-function (PSF)-matched $J_{125^{-}}$and $J_{140}$-band images is used for $z_{850}$-dropouts, ${ }^{14}$ a stack of the PSF-matched $J_{140^{-}}$and $H_{160}$-band images for $Y_{105}$-dropouts, and the $H_{160}$-band image for candidates at $z>8.5$. Their $5 \sigma$ limiting magnitudes are 29.8 $\left(J_{125}+J_{140}\right), 29.7\left(J_{140}+H_{160}\right)$, and $29.5\left(H_{160}\right)$ within filtermatched apertures, which are $0.45-0.50$ arcsec in diameter (Ellis et al. 2013). We use images with a pixel scale of 0.03 arcsec pixel $^{-1}$.

\section{SAMPLES}

We investigate the sizes of $z \sim 7-12$ galaxies based on the $z \sim 7-8$ samples selected by Schenker et al. (2013) and the $z>8.5$ samples whose photometric redshifts from SED fitting analysis are available in McLure et al. (2013; see also Ellis et al. 2013). Here we briefly summarize how these galaxies are selected.

To select star-forming galaxies at $z \sim 7-8$, Schenker et al. (2013) applied the dropout technique, which probes a blue UV spectrum and a spectral break blueward of $\operatorname{Ly} \alpha$ due to intergalactic medium (IGM) absorption. For $z \sim 7 z_{850}$-dropout galaxies, they first required a $3.5 \sigma$ detection in $Y_{105}$ plus one of the other filters which probe longer wavelengths $\left(J_{125}, J_{140}\right.$, or $\left.H_{160}\right)$. Then they applied the two color criteria: $z_{850}-Y_{105}>0.7$ and $Y_{105}-J_{125}<0.4$. Also the following criteria were used: (1) the significance is less than $2.0 \sigma$ in $B_{435}, V_{606}$, and $i_{775}$; (2) the significance is not more than $1.5 \sigma$ in more than one band among $B_{435}, V_{606}$, and $i_{775}$; and (3) $\chi_{\text {opt }}^{2}$ is less than a threshold value. $\chi_{\mathrm{opt}}^{2}$ is defined by $\chi_{\mathrm{opt}}^{2}=\Sigma_{i} \operatorname{SGN}\left(f_{i}\right)\left(f_{i} / \sigma_{i}\right)^{2}$ where $f_{i}$ is the flux in band $i, \sigma_{i}$ is the uncertainty of $f_{i}$, and $\operatorname{SGN}\left(f_{i}\right)$ is 1 if $f_{i}>0$ and -1 if $f_{i}<0$, considering the bands shorter than Ly $\alpha$ (Bouwens et al. 2011b). For $z_{850}$-dropouts, $B_{435}, V_{606}$, and $i_{775}$ are considered, and for $Y_{105}$-dropouts, $B_{435}, V_{606}, i_{775}$, and $z_{850}$ are considered. At or below the $5 \sigma$ limit, they adopted a $\chi_{\text {opt }}^{2}$ upper limit of 2.5, while at the $10 \sigma$ limit they used an upper limit of 5.0. A linear interpolation is used to determine the limit for magnitudes between the $5 \sigma$ and $10 \sigma$ level. For $z \sim 8 Y_{105}$-dropout galaxies, they required a $3.5 \sigma$ detection in $J_{125}$ and one of the other filters which probe longer wavelengths, $J_{140}$ and $H_{160}$. From the detected objects, they selected dropouts which satisfy the two color criteria, $Y_{105}-J_{125}>0.5$ and $J_{125}-H_{160}<0.4$, and the following criteria for the optical data: (1) the significance is less than $2.0 \sigma$ in $B_{435}, V_{606}, i_{775}$, and $z_{850} ;(2)$ the significance is not more than $1.5 \sigma$ in more than one band among $B_{435}, V_{606}, i_{775}$, and $z_{850}$; and (3) $\chi_{\mathrm{opt}}^{2}$ is less than the threshold value. These selection criteria are designed to provide as large a sample of galaxies as possible above redshift 6.5 , while minimizing the effect of contamination (for details, see Section 3.3 of Schenker et al. 2013). By using these selection criteria, Schenker et al. (2013) identified 47 $z_{850}$-dropouts and $27 Y_{105}$-dropouts in the HUDF main field.

\footnotetext{
13 http://udf12.arizona.edu/

14 Although the rest-frame wavelength range covered by the $J_{125}$-band image for $z_{850}$-dropouts is slightly different from that by the $J_{140}$-band image, we confirm that there is no systematic difference between their sizes in these two passbands. We also conduct a similar check for $Y_{105}$-dropouts, using the $J_{140^{-}}$ and $H_{160}$-band images, to confirm that their sizes are likewise consistent within the uncertainties.
} 
McLure et al. (2013) independently searched for galaxies at $z \gtrsim 7$ using the photometric redshift technique. The objects in their catalog with photometric redshifts $z_{\text {photo }} \sim 7-9$ are well matched with the objects in the $z_{850}$ - and $Y_{105}$-dropout catalogs constructed by Schenker et al. (2013).

In addition to the dropout galaxies, we study $z>8.5$ star-forming galaxy candidates reported by Ellis et al. (2013). They located all sources by examining the stack of the final $J_{125^{-}}, J_{140^{-}}$, and $H_{160^{-}}$band images and applied the photometric redshift technique (see also McLure et al. 2013), making use of the full data set obtained by the UDF12 program and the previous programs. They also applied the dropout technique for the master catalog, searching for objects undetected at $2 \sigma$ in both $Y_{105}$ (>31.0 mag) and in a combined ACS image. By both of the two techniques, they have found seven convincing $z>8.5$ candidates.

Morphology measurements for galaxies require a significant detection in not only the central region of sources, but also the outer structures. Recently, Mosleh et al. (2012) reported that, in order to recover the input sizes of their realistic simulations, a signal-to-noise ratio $(\mathrm{S} / \mathrm{N})$ of at least 10 is required. To obtain robust estimates on galaxy morphologies, we set a more strict criterion for $\mathrm{S} / \mathrm{N}$; we analyze our dropouts individually down to $\mathrm{S} / \mathrm{N}$ of 15 . The number of $z_{850}$-dropouts and $Y_{105}$-dropouts with detection greater than $15 \sigma$ in $J_{125}+J_{140}$ and $J_{140}+H_{160}$ (about $28.5 \mathrm{mag}$ in the filter-matched apertures) is 9 and 6 , respectively.

In order to extend our analysis to fainter magnitudes, we divide the samples into three luminosity bins, $L=(0.3-1) L_{z=3}^{*}$, $L=(0.12-0.3) L_{z=3}^{*}$, and $L=(0.048-0.12) L_{z=3}^{*}$, based on their total magnitudes in $J_{125}+J_{140}$ for $z_{850}$-dropouts and $J_{140}+H_{160}$ for $Y_{105}$-dropouts. Since it is difficult to establish reliable total magnitudes for faint sources $(\mathrm{S} / \mathrm{N}<15)$ using GALFIT, we subdivide the galaxies into luminosity bins based on aperture magnitudes which contain $70 \%$ of a point-source flux, after making the appropriate aperture correction to $100 \%$ of anticipated point-source flux (McLure et al. 2013). Tables 1 and 2 list the $z_{850}$-dropouts and $Y_{105}$-dropouts in the luminosity bins, respectively. We make median-stacked images separately for the second and third brightest luminosity bins. The number of $z_{850}$-dropouts ( $Y_{105}$-dropouts) with $L=(0.12-0.3) L_{z=3}^{*}$ is 7 (5), while the number with $L=(0.048-0.12) L_{z=3}^{*}$ is 17 (13). Note that, among the $7 z_{850}$-dropouts (the $5 Y_{105}$-dropouts) in the second brightest luminosity bin, 6 (3) are individually detected at more than $15 \sigma$ in $J_{125}+J_{140}\left(J_{140}+H_{160}\right)$. We do not use stacked images for the objects in the brightest luminosity bin, since the numbers of the objects are small. We also tried stacking objects fainter than $L=0.048 L_{z=3}^{*}$, but they did not provide meaningful size constraints.

Within the $z>8.5$ sample, UDF12-3954-6284 has a relatively high photometric redshift, $z_{\text {photo }}=11.9$, while the others have $z_{\text {photo }}=8.6-9.5$ (Ellis et al. 2013; McLure et al. 2013). Thus, we divide them into two sub-samples: one with UDF123954-6284 and the other with the remaining six objects. The average photometric redshift of the latter subsample is about 9.0. Since most of these objects are quite faint, we make a stack of the $H_{160}$ images of the six $z \simeq 9$ objects, giving a detection with $\mathrm{S} / \mathrm{N} \sim 9$.

Note that the nature of the $z \sim 12$ source is still uncertain, because of its accompanying diffuse morphology (Section 4.2) and its luminosity, particularly given the lack of other detections beyond $z \sim 10.5$ (Ellis et al. 2013). Nevertheless, since no alternative plausible explanation for this object has yet been
Table 1

z850-dropout Galaxies Used in Our Size Analysis

\begin{tabular}{|c|c|c|c|c|}
\hline ID & $\begin{array}{l}\text { R.A. }^{a} \\
\text { (h:m:s) }\end{array}$ & $\begin{array}{l}\text { Decl. }^{\mathrm{a}} \\
\text { (d:m:s) }\end{array}$ & $z_{\text {photo }}{ }^{\mathrm{b}}$ & $\begin{array}{l}M_{\mathrm{UV}}{ }^{\mathrm{c}} \\
(\mathrm{mag})\end{array}$ \\
\hline \multicolumn{5}{|c|}{$L / L_{z=3}^{*}=0.3-1$} \\
\hline UDF12-3746-6328 & $3: 32: 37.46$ & $-27: 46: 32.8$ & 6.4 & -20.54 \\
\hline UDF12-4258-6567 & $3: 32: 42.58$ & $-27: 46: 56.7$ & 7.1 & -20.22 \\
\hline UDF12-4256-7314 & $3: 32: 42.56$ & $-27: 47: 31.4$ & 7.2 & -19.75 \\
\hline \multicolumn{5}{|c|}{$L / L_{z=3}^{*}=0.12-0.3$} \\
\hline UDF12-4219-6278 & $3: 32: 42.19$ & $-27: 46: 27.8$ & 6.7 & -19.13 \\
\hline UDF12-3958-6565 & $3: 32: 39.58$ & $-27: 46: 56.5$ & 6.8 & -19.02 \\
\hline UDF12-3677-7536 & $3: 32: 36.77$ & $-27: 47: 53.6$ & 6.5 & -19.00 \\
\hline UDF12-4105-7156 & $3: 32: 41.05$ & $-27: 47: 15.6$ & 7.1 & -18.98 \\
\hline UDF12-3744-6513 & $3: 32: 37.44$ & $-27: 46: 51.3$ & 6.7 & -18.85 \\
\hline UDF12-3638-7162 & $3: 32: 36.38$ & $-27: 47: 16.2$ & 6.6 & -18.79 \\
\hline UDF12-4057-6436 & $3: 32: 40.57$ & $-27: 46: 43.6$ & 6.8 & -18.71 \\
\hline \multicolumn{5}{|c|}{$L / L_{z=3}^{*}=0.048-0.12$} \\
\hline UDF12-3313-6545 & $3: 32: 33.13$ & $-27: 46: 54.5$ & 7.4 & -18.65 \\
\hline UDF12-4431-6452 & $3: 32: 44.31$ & $-27: 46: 45.2$ & 6.8 & -18.63 \\
\hline UDF12-4160-7045 & $3: 32: 41.60$ & $-27: 47: 04.5$ & 6.7 & -18.53 \\
\hline UDF12-3402-6504 & $3: 32: 34.02$ & $-27: 46: 50.4$ & 7.2 & -18.43 \\
\hline UDF12-4268-7073 & $3: 32: 42.68$ & $-27: 47: 07.3$ & 6.7 & -18.37 \\
\hline UDF12-4239-6243 & $3: 32: 42.39$ & $-27: 46: 24.3$ & 7.3 & -18.32 \\
\hline UDF12-4472-6362 & $3: 32: 44.72$ & $-27: 46: 36.2$ & 6.9 & -18.22 \\
\hline UDF12-4182-6112 & $3: 32: 41.82$ & $-27: 46: 11.2$ & 6.7 & -18.20 \\
\hline UDF12-3853-7519 & $3: 32: 38.53$ & $-27: 47: 51.9$ & 7.1 & -17.95 \\
\hline UDF12-4068-6498 & $3: 32: 40.68$ & $-27: 46: 49.8$ & 7.0 & -17.92 \\
\hline UDF12-3456-6493 & $3: 32: 34.56$ & $-27: 46: 49.3$ & 7.0 & -17.92 \\
\hline UDF12-3975-7451 & $3: 32: 39.75$ & $-27: 47: 45.1$ & 6.9 & -17.90 \\
\hline UDF12-3734-7192 & $3: 32: 37.34$ & $-27: 47: 19.2$ & 6.7 & -17.89 \\
\hline UDF12-3989-6189 & $3: 32: 39.89$ & $-27: 46: 18.9$ & 6.9 & -17.88 \\
\hline UDF12-4384-6311 & $3: 32: 43.84$ & $-27: 46: 31.1$ & 7.2 & -17.85 \\
\hline UDF12-3696-5536 & $3: 32: 36.96$ & $-27: 45: 53.6$ & 6.5 & -17.75 \\
\hline UDF12-3736-6245 & $3: 32: 37.36$ & $-27: 46: 24.5$ & 6.6 & -17.74 \\
\hline
\end{tabular}

Notes.

a Coordinates are in $\mathrm{J} 2000$.

b Photometric redshifts reported by McLure et al. (2013) if available, otherwise $z=6.7$, which corresponds to the peak of the selection function for $z_{850^{-}}$ dropouts.

c Total absolute magnitude measured with the stack of the $J_{125}$ and $J_{140}$ images, whose central wavelength corresponds to $\sim 1570-1780 \AA$ in the rest-frame of the galaxies.

proposed, we analyze this object as a $z \sim 12$ candidate individually.

In our following analysis, we treat the nine $z_{850}$-dropouts and six $Y_{105}$-dropouts with $>15 \sigma$ detections individually, and also the four stacked objects at $z \sim 7-8$. In addition, we analyze the stacked $z \simeq 9$ object and the $z=11.9$ object.

Note that our bright dropouts have been found in the literature as summarized in Table 3 .

\section{SIZES OF GALAXIES AT $z \sim 7-12$}

The Sérsic power law (Sersic 1968) is one of the most frequently used profiles to study galaxy morphology and has the following form:

$$
\Sigma(r)=\Sigma_{e} \exp \left(-b_{n}\left[\left(\frac{r}{r_{e}}\right)^{1 / n}-1\right]\right),
$$

where $\Sigma_{e}$ is the surface brightness at the half-light radius $r_{e}$ and $n$ is the Sérsic index, which is often referred to as the concentration parameter; larger $n$ values denote steeper inner 
Table 2

$Y_{105}$-dropout Galaxies Used in Our Size Analysis

\begin{tabular}{|c|c|c|c|c|}
\hline ID & $\begin{array}{l}\text { R.A. }^{\mathrm{a}} \\
\text { (h:m:s) }\end{array}$ & $\begin{array}{l}\text { Decl. }^{\mathrm{a}} \\
\text { (d:m:s) }\end{array}$ & $z_{\text {photo }}{ }^{b}$ & $\begin{array}{l}M_{\mathrm{UV}}{ }^{\mathrm{c}} \\
(\mathrm{mag})\end{array}$ \\
\hline \multicolumn{5}{|c|}{$L / L_{z=3}^{*}=0.3-1$} \\
\hline UDF12-3880-7072 & $3: 32: 38.80$ & $-27: 47: 07.2$ & 7.7 & -20.29 \\
\hline UDF12-3952-7174 & $3: 32: 39.52$ & $-27: 47: 17.4$ & 7.9 & -20.18 \\
\hline UDF12-4470-6443 & $3: 32: 44.70$ & $-27: 46: 44.3$ & 7.7 & -19.95 \\
\hline \multicolumn{5}{|c|}{$L / L_{z=3}^{*}=0.12-0.3$} \\
\hline UDF12-4314-6285 & $3: 32: 43.14$ & $-27: 46: 28.5$ & 7.3 & -19.27 \\
\hline UDF12-3722-8061 & $3: 32: 37.22$ & $-27: 48: 06.1$ & 7.7 & -19.20 \\
\hline UDF12-3813-5540 & $3: 32: 38.13$ & $-27: 45: 54.0$ & 8.3 & -19.21 \\
\hline UDF12-3780-6001 & $3: 32: 37.80$ & $-27: 46: 00.1$ & 8.1 & -18.84 \\
\hline UDF12-3764-6015 & $3: 32: 37.64$ & $-27: 46: 01.5$ & 8.3 & -18.75 \\
\hline \multicolumn{5}{|c|}{$L / L_{z=3}^{*}=0.048-0.12$} \\
\hline UDF12-3939-7040 & $3: 32: 39.39$ & $-27: 47: 04.0$ & 7.8 & -18.56 \\
\hline UDF12-4474-6450 & $3: 32: 44.74$ & $-27: 46: 45.0$ & 7.8 & -18.41 \\
\hline UDF12-4309-6277 & $3: 32: 43.09$ & $-27: 46: 27.7$ & 8.0 & -18.24 \\
\hline UDF12-4309-6260 & $3: 32: 43.10$ & $-27: 46: 26.0$ & 8.0 & -18.19 \\
\hline UDF12-3463-6472 & $3: 32: 34.63$ & $-27: 46: 47.2$ & 8.0 & -18.14 \\
\hline UDF12-3551-7443 & $3: 32: 35.51$ & $-27: 47: 44.3$ & 8.0 & -18.11 \\
\hline UDF12-4336-6203 & $3: 32: 43.36$ & $-27: 46: 20.3$ & 8.0 & -18.08 \\
\hline UDF12-4240-6550 & $3: 32: 42.40$ & $-27: 46: 55.0$ & 7.8 & -18.01 \\
\hline UDF12-4033-8026 & $3: 32: 40.33$ & $-27: 48: 02.6$ & 7.7 & -17.98 \\
\hline UDF12-4308-6242 & $3: 32: 43.08$ & $-27: 46: 24.2$ & 7.6 & -17.88 \\
\hline UDF12-3934-7256 & $3: 32: 39.34$ & $-27: 47: 25.6$ & 8.0 & -17.85 \\
\hline UDF12-3931-6180 & $3: 32: 39.31$ & $-27: 46: 18.0$ & 7.5 & -17.81 \\
\hline UDF12-3881-6343 & $3: 32: 38.81$ & $-27: 46: 34.3$ & 8.0 & -17.71 \\
\hline
\end{tabular}

\section{Notes.}

${ }^{\text {a }}$ Coordinates are in $\mathrm{J} 2000$.

b Photometric redshifts reported by McLure et al. (2013) if available, otherwise $z=8.0$, which corresponds to the peak of the selection function for $Y_{105}$-dropouts.

c Total absolute magnitude measured with the stack of the $J_{140}$ and $H_{160}$ images, whose central wavelength corresponds to $\sim 1570-1760 \AA$ in the rest-frame of the galaxies.

profiles and highly extended outer wings. The parameter $r_{e}$ is the half-light radius, which holds half of the total flux inside. To make this definition true, the variable $b_{n}$ depends on $n$. We fit the two-dimensional surface brightness profile using the GALFIT software version 3 (Peng et al. 2002, 2010), which convolves a galaxy model profile image with a PSF profile and optimizes the fits using the Levenberg-Marquardt algorithm for $\chi^{2}$ minimization. The output parameters include the centroid coordinates of the objects, their total magnitude, half-light radius, Sérsic index $n$, axis ratio, and position angle. The half-light radius provided by GALFIT is the radius along the semimajor axis, $a$. For each galaxy, we calculate the circularized half-light radius, $r_{e}=a \sqrt{b / a}$, where $b / a$ is the axis ratio. The initial parameters used for profile fitting are provided by SExtractor (Bertin \& Arnouts 1996), and all of the parameters, except for the Sérsic index, $n$, are allowed to vary during the fitting procedure. The Sérsic index $n$ is fixed at 1.0 , which corresponds to the exponential profile. In this case, $b_{n}=1.678$, which is obtained by solving the following equation: $\gamma\left(2 n, b_{n}\right)=\Gamma(2 n) / 2$, where $\gamma$ is the incomplete gamma function, and $\Gamma$ is the gamma function. Although Oesch et al. (2010a) set the Sérsic index $n$ to be 1.5 during their analysis, we confirm that measured sizes show very little difference if we use $n=1.5$. Noise images, required to weight individual pixels in the fit, are taken to be the root mean square (rms) maps generated from variance maps provided by the data reduction. We also use segmentation images which are produced by SExtractor, to mask objects other than the object we are interested in during the profile fitting.

\subsection{Simulations of Systematic Effects}

Low surface brightness in the outskirts of a galaxy may not be correctly measured by GALFIT, leading to systematically low measured half-light radii and/or total magnitudes. The differences in PSF sizes of the filters used in this study ${ }^{15}$ may also cause systematic effects on size measurements. In order to quantify and correct for any such systematic effects, we use the following simulations.

First, we use GALFIT to produce galaxy images whose Sérsic index $n$ is fixed at 1.0, half-light radius $r_{e}$ is randomly chosen between 0.5 and 10.5 pixels, and total magnitude is randomly chosen between 26 and $30 \mathrm{mag}$. Then we convolve them with a PSF image which is a composite of bright and unsaturated stellar objects in the HUDF (Pirzkal et al. 2005). Figure 1 shows the measured PSFs for the $J_{125}+J_{140}, J_{140}+H_{160}$, and $H_{160}$ images. The PSF-convolved galaxy images are inserted into empty regions of the original images before being analyzed in an identical manner to the true galaxy sample.

Figure 2 displays the results of size measurements of our simulated galaxies. The panels show $r_{e}^{\text {(in) }}$ versus $r_{e}^{\text {(out) }}$ for each image at two different magnitude ranges $\left(26<m^{\text {(out) }}<27\right.$ and $27<m^{\text {(out) }}<28$ ). We see that measurements for all images give low systematic offsets for objects with sizes smaller than $\sim 4$ pixels, although at larger sizes the profiles are progressively underestimated as the surface brightness of the objects decreases. The systematics are also seen to be larger for the fainter objects. We also use these simulation results for estimating statistical errors in the measurements.

Figure 3 shows the results for measured total magnitudes compared to input magnitude. This time the results are displayed in two size bins $\left(0.5<r_{e}^{\text {(out) }}<2.5\right.$ pixels, $2.5<r_{e}^{\text {(out) }}<$ 4.5 pixels) for each image. The results for the smaller size bin show that the total measured magnitude is robust down to $\sim 28$ mag. For objects fainter than this the measured magnitude is systematically fainter than the intrinsic value, and the statistical errors increase. The trend is similar for both size bins but the results for larger objects show greater systematic offsets and statistical uncertainties.

Note that the input axis ratio is fixed at $b / a=1$ in the simulations. To investigate the effect of changing this input value on the systematic and statistical uncertainties, we created images with $n=1, r_{e}=2.5$ pixels, which is comparable to the size of the stacked objects and the $z \sim 12$ object, total magnitude $m^{(\mathrm{in})}=26.5-28.5 \mathrm{mag}$, and axis ratios ranging from $b / a=0.2$ to 1 . We convolve these images with the PSF, insert them into blank sky regions, and run GALFIT to measure their best-fit parameters in a similar way to that noted above. The results for the $H_{160}$ image are shown in Figure 4. We find that the output $r_{e}$ is systematically smaller than its input value and that this difference increases for fainter galaxies (by up to $\sim 30 \%$ on average at $m^{(\mathrm{in})}=28.5$ ). However, this bias does not depend significantly on the input axis ratio. Thus, a simple correction for this bias can be made. However, if the axis ratio is smaller than 1, the statistical uncertainties do increase. At the faintest magnitude, the standard deviation increases by $\sim 40 \%$

\footnotetext{
15 http://www.stsci.edu/hst/wfc3/documents/handbooks/currentIHB/ c07_ir07.html
} 

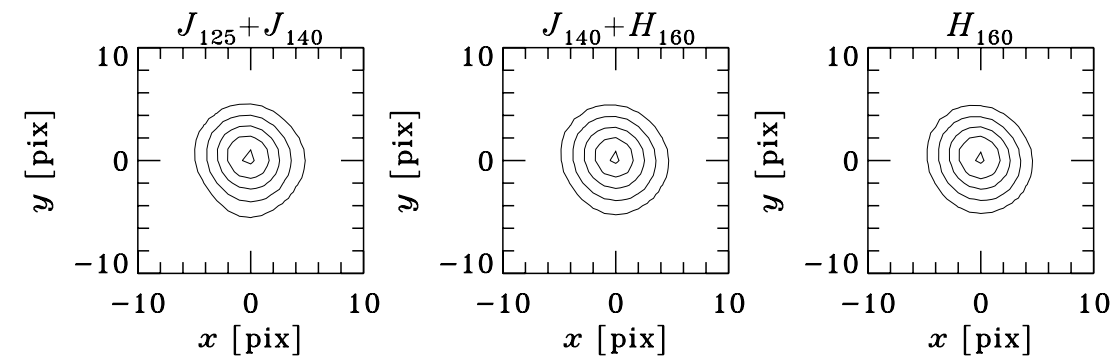

Figure 1. Contours of PSF images in $J_{125}+J_{140}$ (left), $J_{140}+H_{160}$ (middle), and $H_{160}$ (right). The half-light radii of the PSFs are 0.119 arcsec (3.97 pixels) in $J_{125}+J_{140}, 0.124 \operatorname{arcsec}\left(4.14\right.$ pixels) in $J_{140}+H_{160}$, and $0.123 \operatorname{arcsec}\left(4.12\right.$ pixels) in $H_{160}$.

Table 3

Bright $z_{850}$ - and $Y_{105}$-dropout Galaxies in the HUDF Reported in the Literature

\begin{tabular}{|c|c|c|c|c|c|c|c|c|c|c|}
\hline ID & (1) & (2) & (3) & (4) & (5) & (6) & (7) & (8) & (9) & (10) \\
\hline \multicolumn{11}{|c|}{ Bright $z_{850}$-dropouts } \\
\hline UDF12-4258-6567 & UDF-640-1417 & $\ldots$ & UDFz-42566566 & 688 & 1441 & A032 & zD1 & HUDF.z.4444 & UDFz-42566566 & HUDF-658 \\
\hline UDF12-3746-6328 & $\ldots$ & $\ldots$ & $\ldots$ & 837 & 769 & $\ldots$ & $\ldots$ & $\ldots$ & & HUDF-796 \\
\hline UDF12-4256-7314 & UDF-387-1125 & $\ldots$ & UDFz-42577314 & 1144 & 2432 & A008 & zD3 & HUDF.z.6433 & UDFz-42567314 & \\
\hline UDF12-4219-6278 & $\ldots$ & $\ldots$ & & 1464 & 649 & $\ldots$ & $\ldots$ & HUDF.z.2677 & UDFz-42196278 & HUDF-1442 \\
\hline UDF12-3677-7536 & $\ldots$ & $\ldots$ & UDFz-36777536 & 1911 & 2894 & $\ldots$ & $\ldots$ & HUDF.z.7462 & UDFz-36777536 & HUDF-1473 \\
\hline UDF12-4105-7156 & $\ldots$ & $\ldots$ & UDFz-41057156 & 2066 & 2013 & A017 & $\ldots$ & $\ldots$ & $\ldots$ & $\ldots$ \\
\hline UDF12-3958-6565 & $\ldots$ & $\ldots$ & UDFz-39586565 & 1915 & 1445 & A033 & $\ldots$ & $\ldots$ & UDFz-39576564 & HUDF-1995 \\
\hline UDF12-3744-6513 & $\ldots$ & $\ldots$ & UDFz-37446513 & 1880 & 1289 & A040 & $\ldots$ & HUDF.z.4121 & UDFz-37446512 & HUDF-1632 \\
\hline UDF12-3638-7162 & $\ldots$ & $\ldots$ & UDFz-36387163 & 1958 & 2032 & A016 & zD6 & HUDF.z.5659 & UDFz-36377163 & HUDF-1818 \\
\hline \multicolumn{11}{|c|}{ Bright $Y_{105}$-dropouts } \\
\hline UDF12-3880-7072 & UDF-983-964 & HUDF-480 & UDFz-38807073 & 835 & 1768 & A025 & zD2 & HUDF.z.5141 & UDFy-38807071 & HUDF-860 \\
\hline UDF12-4470-6443 & $\ldots$ & $\ldots$ & UDFz-44716442 & 1107 & 1106 & A044 & zD7 & $\ldots$ & UDFy-44706443 & HUDF-1173 \\
\hline UDF12-3952-7174 & $\ldots$ & $\ldots$ & $\ldots{ }^{a}$ & 1422 & 2055 & B041 & $\ldots{ }^{b}$ & $\ldots$ & UDFy-39537174 & \\
\hline UDF12-4314-6285 & $\ldots$ & $\ldots$ & UDFz-43146285 & 1678 & 669 & A060 & zD5 & HUDF.z.2714 & UDFy-43136284 & HUDF-1419 \\
\hline UDF12-3722-8061 & $\ldots$ & $\ldots$ & UDFz-37228061 & 1574 & 3053 & A003 & zD9 & & UDFy-37218061 & HUDF-1660 \\
\hline UDF12-3813-5540 & $\ldots$ & $\ldots$ & UDFy-38135539 & 1721 & 125 & B115 & YD3 & HUDF.YD3 & UDFy-38125539 & HUDF-2003 \\
\hline
\end{tabular}

Bright $z>8.5$ candidates

UDF12-3954-6284

$\ldots$

$\ldots$

$\cdots \quad \cdots$

- $\quad \cdots$

$\cdots$

UDFj-39546284

$\cdots$

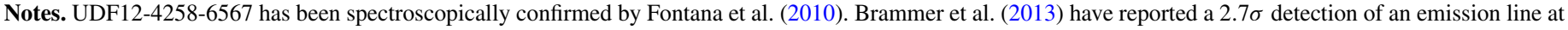
$\sim 1.6 \mu \mathrm{m}$ from UDF12-3954-6284. If it is real, it could be Ly $\alpha$ at $z=12.12$.

a Close to UDFz-39557176.

b Close to zD4.

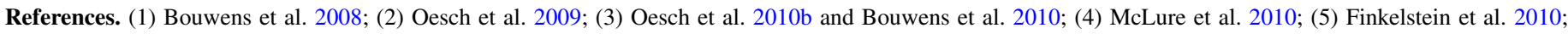

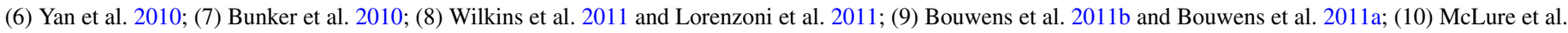
2011.

if the input axis ratio is 0.2 . Although this means the statistical uncertainties in the measured, circularized radii will then be underestimated, the effect is small in most cases.

In summary, our simulations show that GALFIT measurements of half-light radii and total magnitudes are systematically underestimated for faint objects. We correct for systematic effects in the half-light radii and total magnitudes using the measured offsets in Figures 2 and 3, respectively. Note that the errors on $r_{e}$ and total magnitude reported in this paper are also taken from these simulations.

\subsection{GALFIT Measurements}

We perform surface brightness profile fitting for our samples at $z \sim 7-12$, using GALFIT and making use of our simulation results to correct for any systematic effects. We analyze each of the objects with $>15 \sigma$ detections individually $\left(9 z_{850}\right.$-dropouts, $6 Y_{105}$-dropouts), as well as the $z=11.9$ object, which is formally detected at $\sim 8 \sigma$. We extend the analysis to fainter magnitudes using stacked observations. The fainter $z_{850^{-}}$and
$Y_{105}$-dropouts are split into two luminosity bins before stacking $\left(0.12<L / L_{z=3}^{*}<0.3\right.$ and $\left.0.048<L / L_{z=3}^{*}<0.12\right)$, whereas we group all $z \sim 9$ candidates into a single stack.

Figure 5 presents the results of Sérsic profile fitting for the 9 bright $z_{850}$-dropouts. Shown, from left to right, are the $3^{\prime \prime} \times 3^{\prime \prime}$ cut-outs of the original image, the best-fit model produced by GALFIT, the residual images (original image best-fit profile), and the segmentation maps used for masking all the neighboring objects during the profile fitting. Figure 6 similarly shows the results for the 6 bright $Y_{105}$-dropouts. All the objects are cleanly subtracted in the residual images. Note, however that three of the objects (two of the brightest $z_{850^{-}}$ dropouts, UDF12-4258-6567 and UDF12-3746-6327, as well as one of the $Y_{105}$-dropouts, UDF12-3952-7173) are significantly blended with neighboring objects in the original images. ${ }^{16}$ In

\footnotetext{
16 The question of whether each sub-component in these images is associated and therefore represents a source at $z \sim 7-8$ can be addressed by using our rigorous optical non-detection criterion and examining the left panels in Figures 5 and 6.
} 

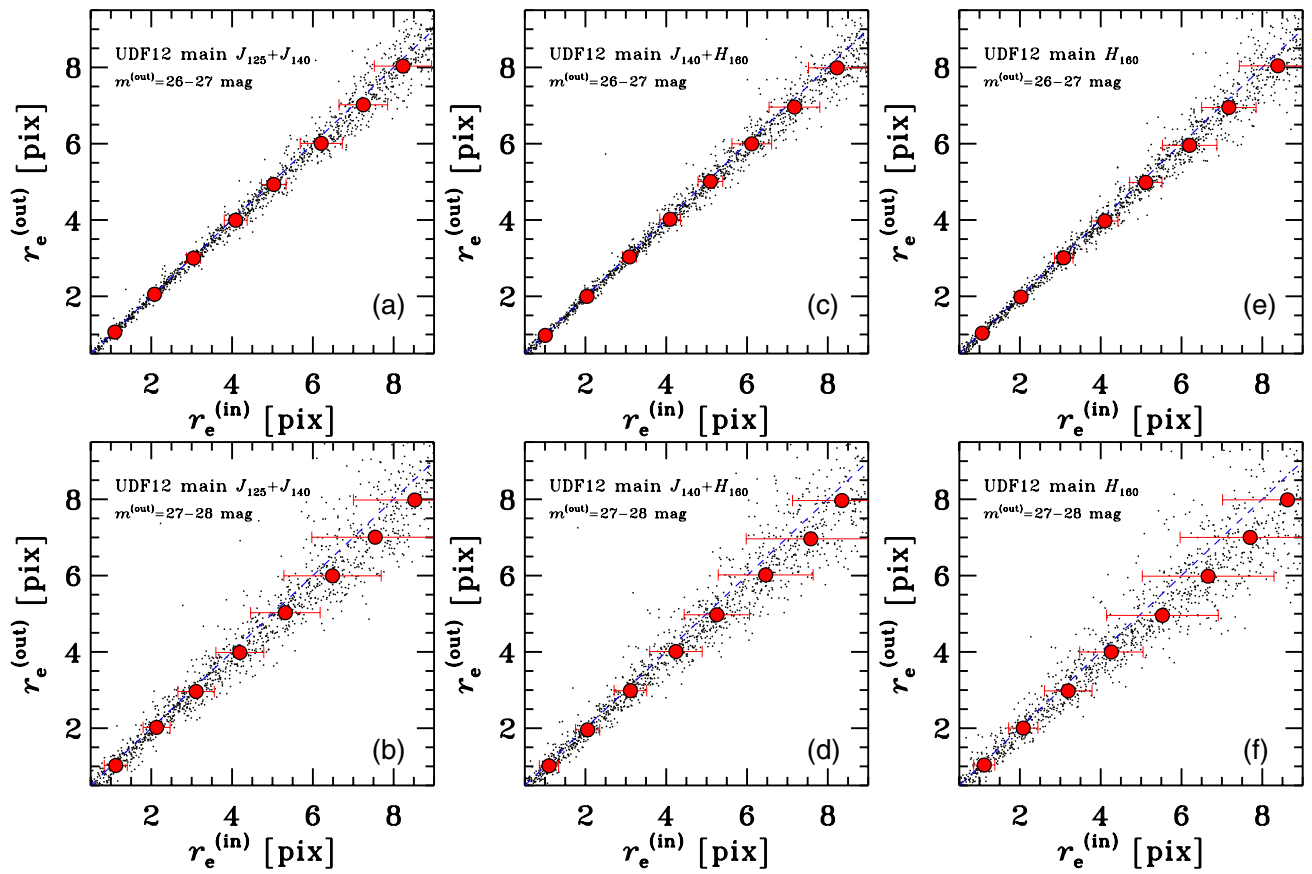

Figure 2. Panels (a) and (b), (c) and (d), and (e) and (f) show the results of our simulations for $z_{850}$-dropouts, $Y_{105}$-dropouts, and $z>8.5$ candidates, respectively. These figures show output radius $r_{e}^{\text {(out) }}$ vs. input half-light radius $r_{e}^{\text {(in) }}$ for a range of output magnitudes, $m^{\text {(out) }}=26-27$ (a, c, e) and 27-28 mag (b, d, f). The red filled circles and the red error bars denote the average value and the relevant rms. The blue dashed line shows the relation of $r_{e}^{(\text {out })}=r_{e}^{\text {(in) }}$.

(A color version of this figure is available in the online journal.)
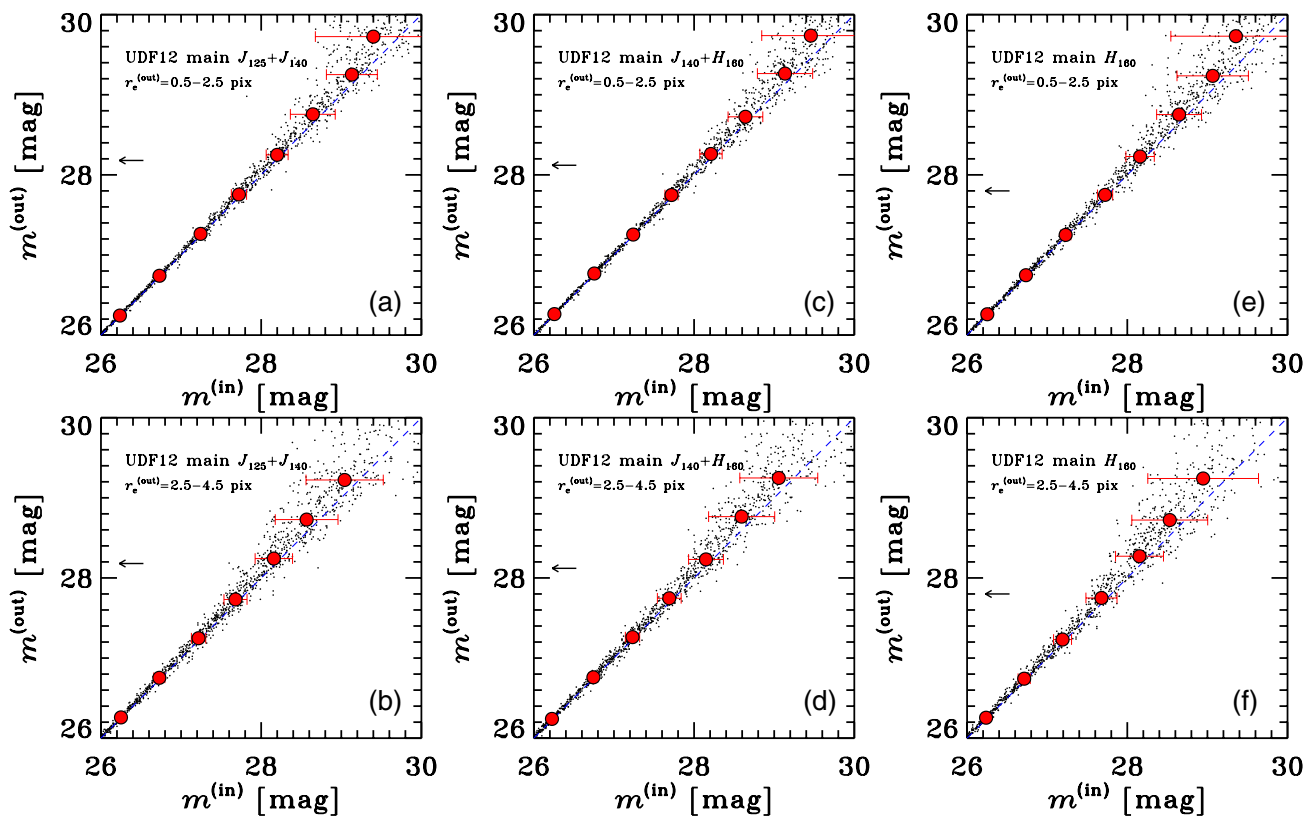

Figure 3. Panels (a) and (b), (c) and (d), and (e) and (f) show the results of our simulations for $z_{850}$-dropouts, $Y_{105}$-dropouts, and $z>8.5$ candidates, respectively. These figures show output magnitude $m^{\text {(out) }}$ vs. input magnitude $m^{\text {(in) }}$ for a range of output half-light radii, $r_{e}^{\text {(out) }}=0.5-2.5$ (a, c, e) and 2.5-4.5 pixels (b, d, f). The red filled circles and the red error bars denote the average value and the relevant rms. The blue dashed line shows the relation of $m^{\text {(out) }}=m^{\text {(in) }}$. The black arrow shows the value of $m^{\text {(out) }}$ (aperture corrected) which corresponds to $\mathrm{S} / \mathrm{N}=15$.

(A color version of this figure is available in the online journal.)

addition, one of the $Y_{105}$-dropouts, UDF12-4470-6442, shows two cores. The uncertainties in the derived profile parameters for these objects will therefore be larger than for other isolated objects.

Although some of the objects in our samples have been analyzed in a previous paper by Oesch et al. (2010a), as those authors do not provide a list of sizes for each candidate explicitly, a direct comparison is not possible. The only exception is
UDFz-42566566. In Section 2 of their paper, they mention that they have treated this object as a two-component source and measured their sizes separately, and reported that the fainter component has a half-light radius of $0.5 \mathrm{kpc}$ and the brighter one has a half-light radius of $0.8 \mathrm{kpc}$. We have selected the fainter component as UDF12-4258-6567, and obtained its halflight radius, $r_{e}=0.48 \pm 0.03 \mathrm{kpc}$, which is in good agreement with the result by Oesch et al. (2010a). Note that the brighter 


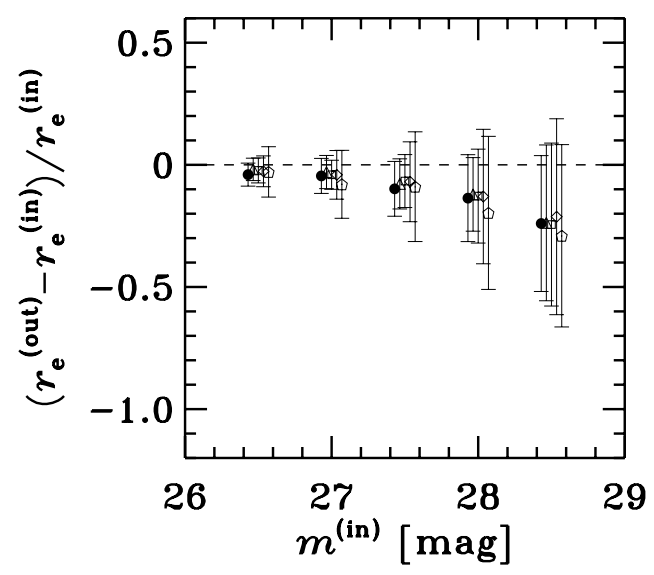

Figure 4. Relative difference between the input and output half-light radii of galaxies in our simulations as a function of input magnitude. The filled circles and the open triangles, squares, diamonds, and pentagons denote the average differences between input half-light radius $r_{e}^{\text {(in) }}$ and output radius $r_{e}^{\text {(out) }}$ with input axis ratio of $b / a=1.0,0.8,0.6,0.4$, and 0.2 , respectively.

component is not included in our sample, due to our rigorous non-detection criterion in the optical.

Figure 7 shows the profile fitting result for the $z \sim 12$ object, UDF12-3954-6284. Since the magnitude of the $z \sim 12$ object in $H_{160}$ measured with $0^{\prime \prime} .50$ diameter aperture is $29.2 \mathrm{mag}$, corresponding to $\mathrm{S} / \mathrm{N} \sim 8$, the profile fitting for this object is quite challenging. Actually, the best-fit model galaxy profile seems more elongated than that in the original image shown in Figure 7, which would overestimate of its total magnitude. At least, the residual image in Figure 7 does not clearly show any noticeable residuals around the central position, although the uncertainties of the fitting parameters are relatively large as inferred from the moderate $\mathrm{S} / \mathrm{N}$ ratio. If we measure the curve of growth for this object, using progressively larger circular apertures, we find that the magnitude saturates at $28.8 \mathrm{mag}$ within an aperture diameter $\sim 0$.' 45 . We also find by this robust method that the half-light of the source is covered by about 0 !'35 diameter aperture, and after considering the PSF broadening effect, we obtain its half-light radius, $r_{\mathrm{hl}}=0.45 \mathrm{kpc}$, which is consistent with the GALFIT measurement within $\sim 1 \sigma$, and is also nearly equal to the value reported by Bouwens et al. (2013), $\sim 0.5 \mathrm{kpc}$.

Additionally, we note that this object has an unusual morphology. It is visually confirmed that the $z \sim 12$ object has a diffuse filamentary structure stretching from northeast to southwest, although the significance is very low. This has been already mentioned very recently by Bouwens et al. (2013). Figure 8 shows the cutout $H_{160}$ images of this object from various subsets and the full data. The diffuse structure is seen in the full $(2009+2012)$ data and in the 2009 data. The $2009 \mathrm{~b}$ cutout also shows a low-S/N filament, and the 2009a cutout has a similar pattern along the same direction. If this diffuse filament is indeed associated with the source at $z \sim 12$, it corresponds to its bright UV continuum and/or $\operatorname{Ly} \alpha$, which would suggest that this object is experiencing a major merger event, leading to their high star formation activity. This star formation enhancement may explain the visibility of such a high-redshift galaxy.

Figures 9 and 10 show profile fitting results for the stacked $z_{850}$-dropouts and $Y_{105}$-dropouts, respectively, whose UV luminosities are $L=(0.12-0.3) L_{z=3}^{*}$ (top) and $L=$ $(0.048-0.12) L_{z=3}^{*}$ (bottom). Also shown in Figure 11 is the profile fitting result for the stacked $z \simeq 9$ candidates. Note that we

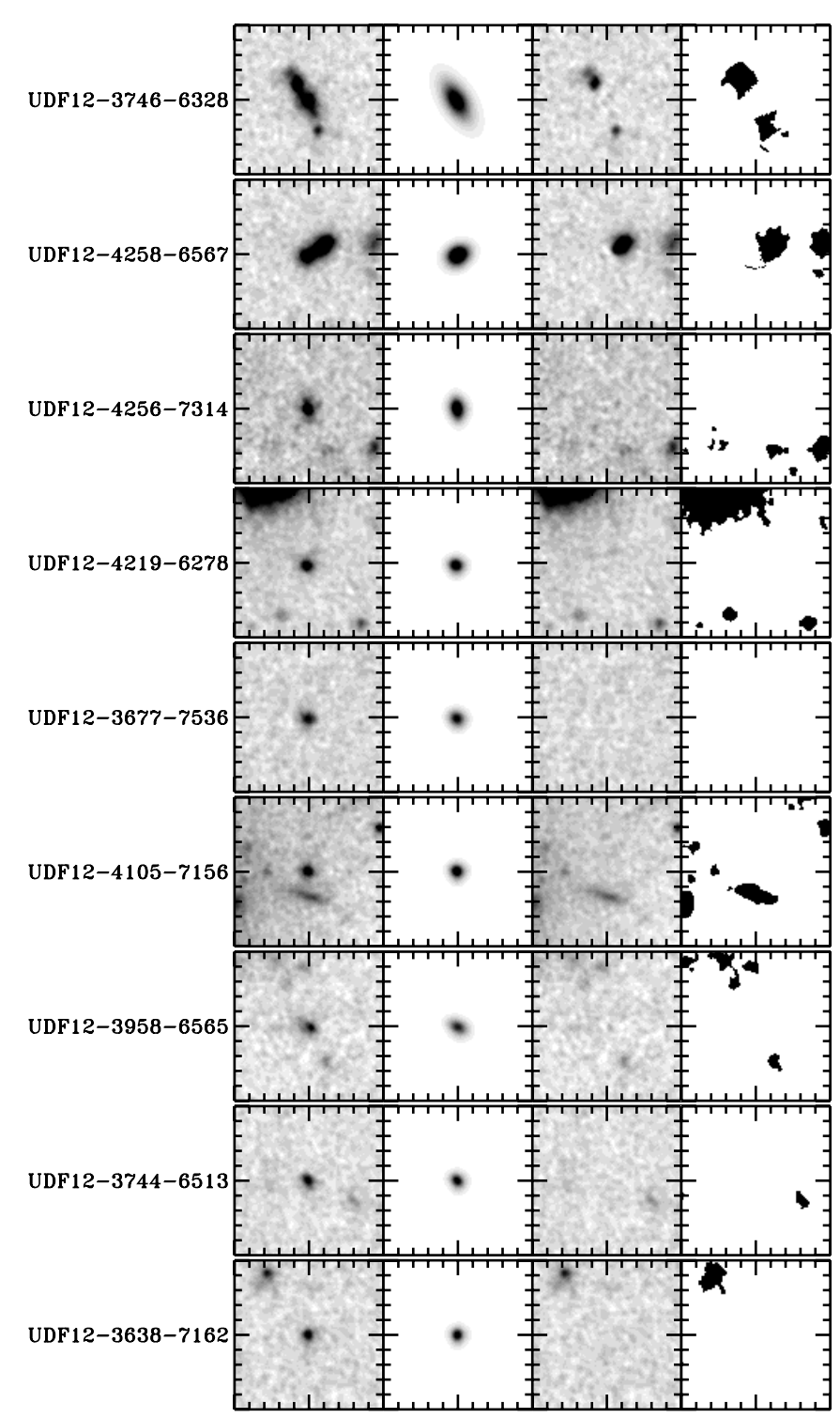

Figure 5. Sérsic profile fitting results for bright $z_{850}$-dropouts found in the HUDF main field. Shown, from left to right, are the $3^{\prime \prime} \times 3^{\prime \prime}$ cut-outs of the original image, the best-fit model profile images, the residual images which are made by subtracting the best-fit images from the original ones, and the segmentation maps used for masking all the neighboring objects during the profile fitting.

also make averaged (not median-stacked) images and perform profile fitting using GALFIT, which yields similar fitting results, although for some of the average stacks, GALFIT does not provide a reasonable fit due to severe confusion with neighboring objects.

In the brightest luminosity bin, $L=(0.3-1) L_{z=3}^{*}$, we do not perform a stacking analysis, since the numbers of the dropouts are small ( 3 for $z_{850}$-dropouts and 3 for $Y_{105}$-dropouts) and the stacked images are significantly confused by neighboring objects. Instead, we calculate their average sizes and magnitudes; $r_{e}=0.64 \pm 0.32 \mathrm{kpc}$ and $M_{\mathrm{UV}}=$ $-20.17 \pm 0.32 \mathrm{mag}(z \sim 7)$ and $r_{e}=0.65 \pm 0.28 \mathrm{kpc}$ and $M_{\mathrm{UV}}=-20.14 \pm 0.14 \mathrm{mag}(z \sim 8)$.

The best-fit parameters are summarized in Table 4 for the $z_{850}$-dropouts, Table 5 for the $Y_{105}$-dropouts, and Table 6 for the $z>8.5$ candidates. The weighted means of half-light radii for the $z_{850}$-dropouts and $Y_{105}$-dropouts with $L=(0.05-1) L_{z=3}^{*}$ are 
Table 4

Surface Brightness Profile Fitting Results for Bright $z_{850}$-dropouts

\begin{tabular}{|c|c|c|c|c|c|}
\hline Object ID & $\begin{array}{c}m_{\mathrm{UV}}^{(\mathrm{ap}) \mathrm{a}} \\
(\mathrm{mag})\end{array}$ & $n^{\mathrm{b}}$ & $\begin{array}{l}m_{\mathrm{UV}}{ }^{\mathrm{c}} \\
(\mathrm{mag})\end{array}$ & $\begin{array}{l}M_{\mathrm{UV}}^{\mathrm{d}} \\
(\mathrm{mag})\end{array}$ & $\begin{array}{c}r_{e} \mathrm{e}^{\mathrm{e}} \\
(\mathrm{kpc})\end{array}$ \\
\hline \multicolumn{6}{|c|}{$\mathrm{S} / \mathrm{N}>15, L / L_{z=3}^{*}=0.3-1$} \\
\hline UDF12-3746-6328 & 27.38 & 1.0 & $26.25 \pm 0.06$ & $-20.54 \pm 0.06$ & $1.09 \pm 0.09$ \\
\hline UDF12-4258-6567 & 27.41 & 1.0 & $26.74 \pm 0.05$ & $-20.22 \pm 0.05$ & $0.48 \pm 0.03$ \\
\hline UDF12-4256-7314 & 27.73 & 1.0 & $27.23 \pm 0.05$ & $-19.75 \pm 0.05$ & $0.36 \pm 0.05$ \\
\hline \multicolumn{6}{|c|}{$\mathrm{S} / \mathrm{N}>15, L / L_{z=3}^{*}=0.12-0.3$} \\
\hline UDF12-4219-6278 & 28.09 & 1.0 & $27.74 \pm 0.10$ & $-19.13 \pm 0.10$ & $0.25 \pm 0.07$ \\
\hline UDF12-3677-7536 & 28.22 & 1.0 & $27.82 \pm 0.11$ & $-19.00 \pm 0.11$ & $0.37 \pm 0.09$ \\
\hline UDF12-4105-7156 & 28.30 & 1.0 & $27.98 \pm 0.13$ & $-18.98 \pm 0.13$ & $0.20 \pm 0.08$ \\
\hline UDF12-3958-6565 & 28.33 & 1.0 & $27.87 \pm 0.11$ & $-19.02 \pm 0.11$ & $0.40 \pm 0.10$ \\
\hline UDF12-3744-6513 & 28.38 & 1.0 & $28.02 \pm 0.13$ & $-18.85 \pm 0.13$ & $0.15 \pm 0.09$ \\
\hline UDF12-3638-7162 & 28.41 & 1.0 & $28.05 \pm 0.14$ & $-18.79 \pm 0.14$ & $0.10 \pm 0.08$ \\
\hline \multicolumn{6}{|c|}{ Stack } \\
\hline UDF12z-stack1 $\left(L / L^{*}=0.12-0.3\right)$ & 28.33 & 1.0 & $27.94 \pm 0.12$ & $-18.93 \pm 0.12$ & $0.28 \pm 0.09$ \\
\hline UDF12 $z$-stack2 $\left(L / L^{*}=0.048-0.12\right)$ & 29.20 & 1.0 & $28.73 \pm 0.26$ & $-18.19 \pm 0.26$ & $0.35 \pm 0.14$ \\
\hline
\end{tabular}

Notes.

a Measured in 0.'46 diameter aperture with the stack of the $J_{125}$ and $J_{140}$ images.

${ }^{\mathrm{b}}$ Sérsic index. This is fixed, not measured.

c Total magnitude measured by GALFIT. The systematic effect is considered.

d Total absolute magnitude calculated using $z_{\text {photo }}$ if available, otherwise $z=6.7$, which corresponds to the peak of the selection function for $z 850$-dropouts.

${ }^{\mathrm{e}}$ Circularized half-light radius $r_{e}=a \sqrt{b / a}$, where $a$ is the radius along the major axis, and $b / a$ is the axis ratio.

Table 5

Surface Brightness Profile Fitting Results for Bright $Y_{105}$-dropouts

\begin{tabular}{|c|c|c|c|c|c|}
\hline Object ID & $\begin{array}{c}m_{\mathrm{UV}}^{(\mathrm{ap}) \mathrm{a}} \\
(\mathrm{mag})\end{array}$ & $n^{\mathrm{b}}$ & $\begin{array}{l}m_{\mathrm{UV}}{ }^{\mathrm{c}} \\
(\mathrm{mag})\end{array}$ & $\begin{array}{l}M_{\mathrm{UV}}^{\mathrm{d}} \\
(\mathrm{mag})\end{array}$ & $\begin{array}{c}r_{e} \mathrm{e} \\
(\mathrm{kpc})\end{array}$ \\
\hline \multicolumn{6}{|c|}{$\mathrm{S} / \mathrm{N}>15, L / L_{z=3}^{*}=0.3-1$} \\
\hline UDF12-3879-7072 & 27.21 & 1.0 & $26.80 \pm 0.03$ & $-20.29 \pm 0.03$ & $0.34 \pm 0.02$ \\
\hline UDF12-4470-6443 & 27.69 & 1.0 & $27.13 \pm 0.08$ & $-19.95 \pm 0.08$ & $0.59 \pm 0.08$ \\
\hline UDF12-3952-7174 & 28.10 & 1.0 & $26.95 \pm 0.10$ & $-20.18 \pm 0.10$ & $1.03 \pm 0.19$ \\
\hline \multicolumn{6}{|c|}{$\mathrm{S} / \mathrm{N}>15, L / L_{z=3}^{*}=0.12-0.3$} \\
\hline UDF12-4314-6285 & 28.10 & 1.0 & $27.73 \pm 0.08$ & $-19.27 \pm 0.08$ & $0.33 \pm 0.06$ \\
\hline UDF12-3722-8061 & 28.28 & 1.0 & $27.89 \pm 0.10$ & $-19.20 \pm 0.10$ & $0.19 \pm 0.06$ \\
\hline UDF12-3813-5540 & 28.33 & 1.0 & $27.99 \pm 0.11$ & $-19.21 \pm 0.11$ & $0.10 \pm 0.05$ \\
\hline \multicolumn{6}{|c|}{ Stack } \\
\hline UDF12y-stack1 $\left(L / L^{*}=0.12-0.3\right)$ & 28.37 & 1.0 & $27.98 \pm 0.11$ & $-19.15 \pm 0.11$ & $0.31 \pm 0.08$ \\
\hline UDF12y-stack2 $\left(L / L^{*}=0.048-0.12\right)$ & 29.47 & 1.0 & $28.90 \pm 0.29$ & $-18.23 \pm 0.29$ & $0.36 \pm 0.13$ \\
\hline
\end{tabular}

Notes.

${ }^{a}$ Measured in 0'.50 diameter aperture with the stack of the $J_{140}$ and $H_{160}$ images.

${ }^{\mathrm{b}}$ Sérsic index. This is fixed, not measured.

${ }^{c}$ Total magnitude measured by GALFIT. The systematic effect is considered.

d Total absolute magnitude calculated using $z_{\text {photo }}$ if available, otherwise $z=8.0$, which corresponds to the peak of the selection function for $Y_{105}$-dropouts.

${ }^{\mathrm{e}}$ Circularized half-light radius $r_{e}=a \sqrt{b / a}$, where $a$ is the radius along the major axis, and $b / a$ is the axis ratio.

$0.32 \pm 0.07 \mathrm{kpc}$ and $0.34 \pm 0.07 \mathrm{kpc}$, respectively. In the next section, we present the size-luminosity relation, and investigate the redshift evolution of galaxy sizes and SFR surface densities based on these results.

\section{RESULTS AND DISCUSSION}

Our measurements of half-light radii in Tables 4-6 show very small values, typically $\lesssim 0.5 \mathrm{kpc}$ (see also filled symbols in Figure 12). The average half-light radii of the dropouts are only $\simeq 0.3-0.4 \mathrm{kpc}$ at $z \sim 7-8$ (Section 4.2) and at $z>8.5$ (Table 6). The half-light radius of our $z \sim 12$ candidate is also remarkably small, $0.32 \pm 0.14 \mathrm{kpc}$. Even including the $1 \sigma$ uncertainties, these half-light radii are, coincidentally, just as large as those of giant molecular associations (GMAs) with a mass of $\sim 10^{7} M_{\odot}$ found in the local universe (e.g., Vogel et al. 1988; Rand \& Kulkarni 1990; Tosaki et al. 2007).

\subsection{Size-Luminosity Relation of Galaxies at $z \sim 7-8$}

We investigate the relation between size and luminosity, i.e., the size-luminosity relation, at each redshift. Figure 12 presents the size-luminosity relation for our $z_{850}$-dropout and $Y_{105}$-dropout galaxies at $z \sim 7-8$. Our $z>8.5$ galaxy candidates are not shown, because we cannot constrain the relation with only two measurements (one from an individual object and one 
Table 6

Surface Brightness Profile Fitting Results for $z>8.5$ Candidates

\begin{tabular}{lccccccc}
\hline \hline Object ID & $\begin{array}{c}\text { R.A. } \\
(\mathrm{h}: \mathrm{m}: \mathrm{s})\end{array}$ & $\begin{array}{c}\text { Decl. } \\
(\mathrm{d}: \mathrm{m}: \mathrm{s})\end{array}$ & $\begin{array}{c}m_{\mathrm{UV}}^{\mathrm{ap}} \mathrm{b} \\
(\mathrm{mag})\end{array}$ & $n^{\mathrm{c}}$ & $\begin{array}{c}m_{\mathrm{UV}} \mathrm{d}^{\mathrm{d}} \\
(\mathrm{mag})\end{array}$ & $\begin{array}{c}M_{\mathrm{UV}} \\
(\mathrm{mag})\end{array}$ & $\begin{array}{c}r_{e} \\
(\mathrm{kpc})\end{array}$ \\
\hline UDF12-3954-6284 & $3: 32: 39.54$ & $-27: 46: 28.4$ & 29.24 & 1.0 & $28.47 \pm 0.25$ & $-20.41 \pm 0.25$ \\
\hline \multicolumn{7}{c}{ Stack } \\
\hline UDF12hz-stack & & 29.69 & 1.0 & $29.11 \pm 0.49$ & $-18.21 \pm 0.49$ \\
\hline
\end{tabular}

Notes.

${ }^{\text {a }}$ Coordinates are in $\mathrm{J} 2000$.

${ }^{\mathrm{b}}$ Measured in 0 '.50 diameter aperture with the $H_{160}$ image.

c Sérsic index. This is fixed, not measured.

d Total magnitude measured by GALFIT. The systematic effect is considered. For UDF12-3954-6284, this might be overestimated, since the best-fit model galaxy profile seems more elongated than that in the original image shown in Figure 7. If we measure the curve of growth for this source, the magnitude saturates at $28.8 \mathrm{mag}$ (see Section 4.2).

${ }^{\mathrm{e}}$ Total absolute magnitude. We calculate it with $z_{\text {photo }}=11.9$ for UDF12-3954-6284, considering IGM absorption shortward of its Ly $\alpha$ wavelength. We use the average photometric redshift, $z_{\text {photo }}=9.0$ for the stacked object.

${ }^{\mathrm{f}}$ Circularized half-light radius $r_{e}=a \sqrt{b / a}$, where $a$ is the radius along the major axis, and $b / a$ is the axis ratio.

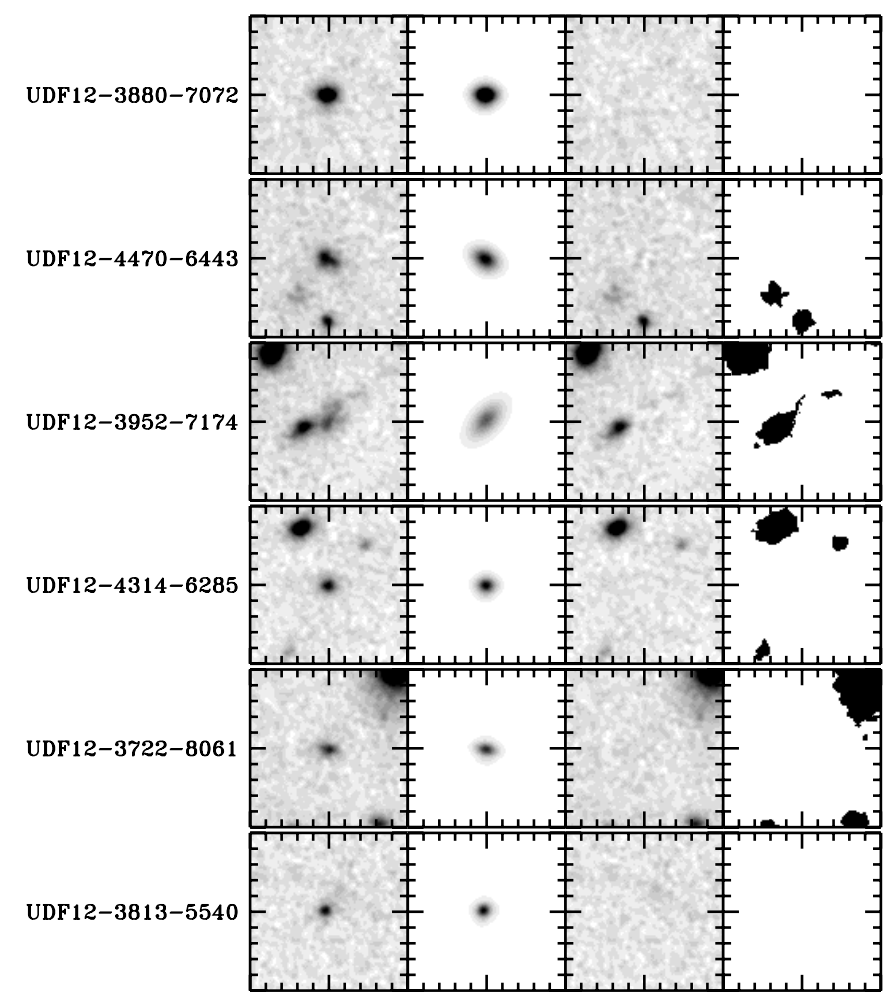

Figure 6. Sérsic profile fitting results for bright $Y_{105}$-dropouts found in the HUDF main field. Shown, from left to right, are the $3^{\prime \prime} \times 3^{\prime \prime}$ cut-outs of the original image, the best-fit model profile images, the residual images which are made by subtracting the best-fit images from the original ones, and the segmentation maps used for masking all the neighboring objects during the profile fitting.

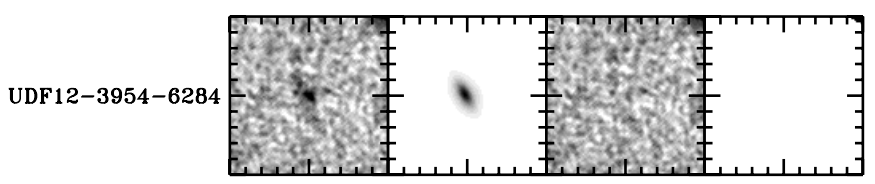

Figure 7. Sérsic profile fitting results for the $z \sim 12$ source, UDF12-3954-6284. Shown, from left to right, are the $3^{\prime \prime} \times 3^{\prime \prime}$ cut-outs of the original image, the best-fit model profile images, the residual images which are made by subtracting the best-fit images from the original ones, and the segmentation maps used for masking all the neighboring objects during the profile fitting.

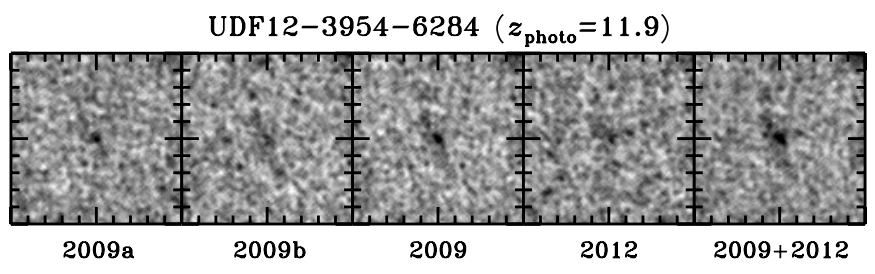

Figure 8. $3^{\prime \prime} \times 3^{\prime \prime}$ cut-outs of the $z \sim 12$ source UDF12-3954-6284 from various subsets of the WFC3/IR $H_{160}$-band observations. From left to right, the first half of the 2009 dataset, the second half of the 2009 dataset, the full 53-orbit 2009 dataset, the 26-orbit 2012, and the full 84-orbit dataset (including 2009, 2012, and other exposures in this field).

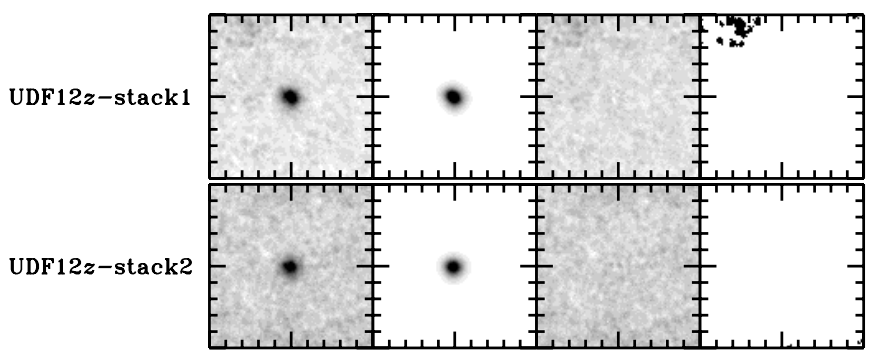

Figure 9. Same as Figure 5, except that the objects are the stacked $z 850$-dropouts, whose UV luminosities are $L=(0.12-0.3) L_{z=3}^{*}$ (top) and $L=(0.048-0.12) L_{z=3}^{*}$ (bottom).

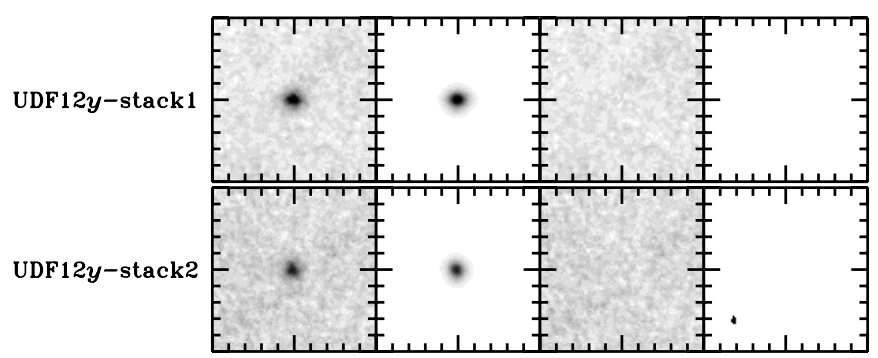

Figure 10. Same as Figure 9, except that the objects are the stacked $Y_{105}$-dropouts.

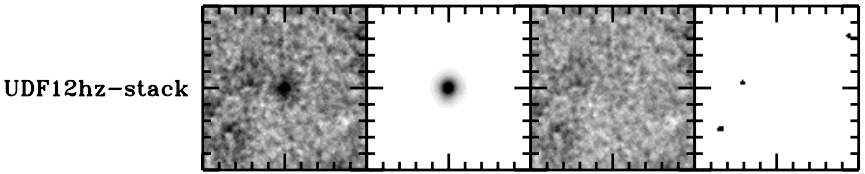

Figure 11. Same as Figure 9, except that the object is the stacked $z \sim 9$ object. 


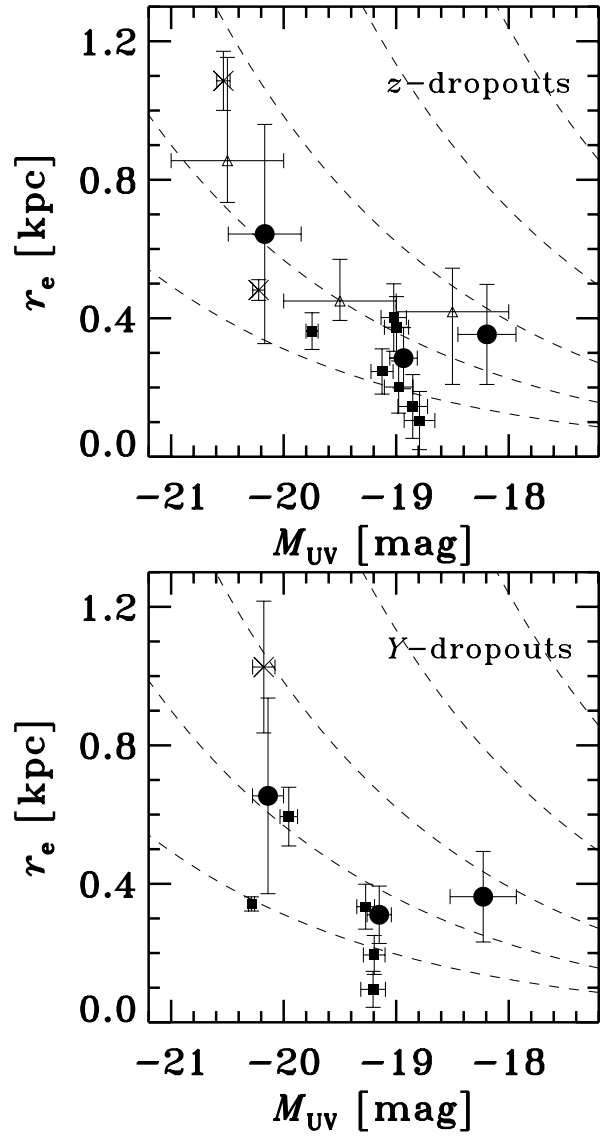

Figure 12. Size-luminosity relation for $z_{850}$-dropouts (top) and $Y_{105}$-dropouts (bottom). The filled circles correspond to the stacked objects with UV luminosities of $L=(0.12-0.3) L_{z=3}^{*}, L=(0.048-0.12) L_{z=3}^{*}$, and the averaged values of the objects with $L=(0.3-1) L_{z=3}^{*}$. The filled squares show the bright objects detected at $>15 \sigma$ without any blending with neighboring sources, while the crosses show the objects detected in more than $15 \sigma$ and blended with a neighboring source. The open triangles in the top panel are taken from Grazian et al. (2012). The dashed curves in each figure correspond to a constant star formation rate density $\Sigma_{\mathrm{SFR}}\left[M_{\odot} \mathrm{yr}^{-1} \mathrm{kpc}^{-2}\right]=0.1,0.3,1,3,10$ from top to bottom.

from the stack). In Figure 12, fainter galaxies have a smaller half-light radius than brighter galaxies. This trend is the same as those of local galaxies (de Jong \& Lacey 2000) as well as high$z$ dropout galaxies studied by Grazian et al. (2012). Because the luminosity of a galaxy depends on two physical quantities (surface brightness and size), one needs to clarify which quantity is dominant in shaping the size-luminosity relation. We define SFR surface density, $\Sigma_{\mathrm{SFR}}$, as the average SFR in a circle whose radius is $r_{e}$,

$$
\Sigma_{\mathrm{SFR}}\left[M_{\odot} \mathrm{yr}^{-1} \mathrm{kpc}^{-2}\right] \equiv \frac{\mathrm{SFR} / 2}{\pi r_{e}^{2}} .
$$

A multiplicative factor of $1 / 2$ is applied since the SFR is estimated from the total magnitude in the rest-frame UV. In the case that dust extinction is negligible, a rest-frame UV luminosity density approximately correlates with an SFR (Kennicutt 1998a),

$$
\operatorname{SFR}\left(M_{\odot} \mathrm{yr}^{-1}\right)=1.4 \times 10^{-28} L_{v}\left(\mathrm{erg} \mathrm{s}^{-1} \mathrm{~Hz}^{-1}\right) .
$$

From Equations (2) and (3), we obtain

$$
M_{\mathrm{UV}}=-2.5 \log \left(\frac{\Sigma_{\mathrm{SFR}} r_{e}^{2}}{1.4 \times 10^{-28} \cdot 2 \cdot(10 \mathrm{pc}(\mathrm{cm}))^{2}}\right)-48.6 .
$$
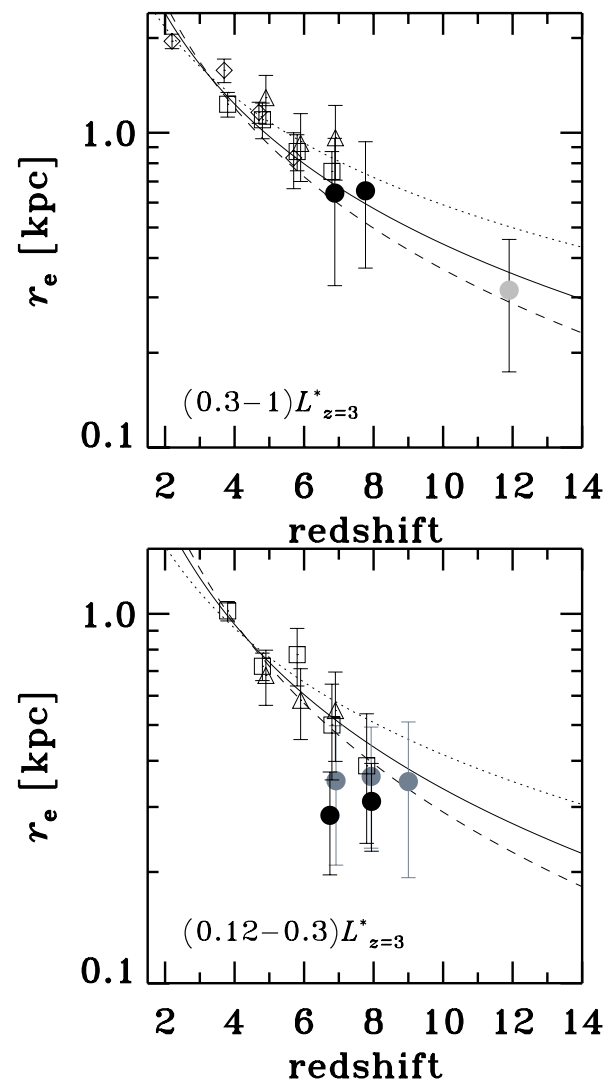

Figure 13. Top: evolution of the half-light radius across the redshift range from $z \sim 2-12$ in $(0.3-1) L_{z=3}^{*}$. The filled circles show the average sizes of our $z_{850}$-dropouts and $Y_{105}$-dropouts, and the size of the $z \sim 12$ object. The open symbols are taken from the literature; the open squares and triangles are dropout galaxies taken from Oesch et al. (2010a), the open diamonds are from Bouwens et al. (2004). After excluding the sample overlaps, we fit simple functions of $(1+z)^{s}$ with the data in both luminosity bins, and obtain $s=-1.30_{-0.14}^{+0.12}$, which is shown as the solid line. The dotted and dashed lines correspond to the case of $s=-1.0$ and -1.5 , respectively. Bottom: evolution of the half-light radius across the redshift range from $z \sim 4-8$ in $(0.12-0.3) L_{z=3}^{*}$. The open and filled black symbols denote the same as those in the top panel. The gray filled circles are dropout galaxies in the fainter luminosity bin, $(0.048-0.12) L_{z=3}^{*}$. The solid, dotted, and dashed lines are the same as those in the top figure.

In Figure 12, we show constant SFR surface densities of $\Sigma_{\mathrm{SFR}}=0.1,0.3,1,3$, and $10\left(M_{\odot} \mathrm{yr}^{-1} \mathrm{kpc}^{-2}\right)$ with dashed lines. We find that most of the individual galaxies and stacked galaxies fall in the range of $\Sigma_{\mathrm{SFR}} \simeq 1-10$ within their uncertainties. These results indicate that both bright and faint $z \sim 7-8$ galaxies have similar SFR surface densities of $\Sigma_{\mathrm{SFR}} \simeq 1-10$, and that the size-luminosity relation at each redshift is mainly determined by the size of galaxies that have a similar SFR surface density.

\subsection{Size Evolution and Its Implications for Galaxy Formation}

We then investigate the size evolution. Since the half-light radius depends on luminosity, as displayed by the size-luminosity relation, we carefully compare the half-light radii of our dropout galaxies within a fixed magnitude range. Figure 13 presents the average half-light radius as a function of redshift for our dropout galaxies at $z \sim 7-12$ with $(0.3-1) L_{z=3}^{*}$ and $(0.12-0.3) L_{z=3}^{*}$, together with dropout galaxies at $z \sim 4-8$ taken from the literature. Our measurements of average half-light radii are consistent with those from the previous studies at $z \sim 7-8$, where we see overlap with previous measurements. Note that the sizes of our $z \sim 7$ galaxies in the fainter luminosity bin are marginally 
discrepant with that estimated by Oesch et al. (2010a). As discussed earlier, unfortunately a galaxy-by-galaxy comparison is not possible. There are two possible reasons for this difference. First, the current paper deals with UDF12 data of much greater depth than that analyzed by Oesch et al. (2010a) who used only the first epoch of the HUDF09 survey. Second, our sample selection criteria are somewhat different as discussed by Schenker et al. (2013). Figure 13 indicates that the average half-light radius decreases with redshift from $z \sim 4$ to 8, which is consistent with the claims of previous studies (e.g., Ferguson et al. 2004; Bouwens et al. 2004; Hathi et al. 2008; Oesch et al. 2010a).

UDF12 provides us with the deepest ever near-infrared images of the HUDF, allowing our study to extend the dynamic range of redshift in the size evolution analysis from $z \sim 8$ to $z \sim 12$, and identifies that the decreasing trend holds up to $z \sim 12$ as shown in Figure 13, if the putative $z \sim 12$ source is real. Although the statistical uncertainty of the measurements is large, the half-light radius of $z \sim 12$ is $r_{e}=0.32 \pm 0.14$ $\mathrm{kpc}$ in the luminosity bin of $(0.3-1) L_{z=3}^{*}$, which is significantly smaller than that of $z \sim 6$ by a factor of three. Note that we can only plot the half-light radius at $z \sim 12$ on the panel of $(0.3-1) L_{z=3}^{*}$ in Figure 13, because there are no $z \sim 12$ galaxies with $(0.12-0.3) L_{z=3}^{*}$ in the UDF12 data. Similarly, our stack of $z>8.5$ galaxies have a luminosity fainter than $(0.12-0.3) L_{z=3}^{*}$, which is too faint to be compared with the baseline of the average half-light radii at $z \sim 4-6$. However, our results of $z>7$ galaxies at these faint magnitudes, which are shown as gray filled circles in the bottom panel of Figure 13, are consistent with the decreasing trend of the half-light radius, albeit with large errors.

This decreasing trend may be explained by the evolution of the host dark halo radius. According to the analytic model in the hierarchical structure formation framework of $\Lambda \mathrm{CDM}$ (see, e.g., Mo et al. 1998; Mo \& White 2002; Ferguson et al. 2004), the virial radius of a dark matter halo is given by

$$
r_{\mathrm{vir}}=\left(\frac{G M_{\mathrm{vir}}}{100 H(z)^{2}}\right)^{1 / 3},
$$

where $H(z)$ is the Hubble parameter and $M_{\text {vir }}$ is the virial mass of the halo. The virial radius is also expressed as a function of the circular velocity of the dark halo by

$$
r_{\mathrm{vir}}=\frac{v_{\mathrm{vir}}}{10 H(z)}
$$

Since $H(z)$ is approximated by $\sim(1+z)^{3 / 2}$ in a flat universe at high redshifts, the redshift evolution of the virial radius is $r_{\text {vir }} \propto H(z)^{-2 / 3} \sim(1+z)^{-1}$ for constant halo mass and $r_{\mathrm{vir}} \propto H(z)^{-1} \sim(1+z)^{-1.5}$ for constant velocity.

Figure 13 shows the radius-redshift relation of dark matter halos for the case of constant halo mass and constant velocity. Previous studies investigating the radius-redshift relation in the redshift range $4<z<8$ reach two different conclusions. Bouwens et al. $(2004,2006)$ claim that the relation is roughly $(1+z)^{-1}$, suggestive that the sizes of disks scale with constant halo mass, while Ferguson et al. (2004) and Hathi et al. (2008) argue that $(1+z)^{-1.5}$, i.e., the constant velocity case, is preferable. We fit the radius-redshift relation over a wider range of redshift (extending to $z \sim 12$ ) with a function of $(1+z)^{s}$, where $s$ is a free parameter. We take into account our size measurements: for the brighter bin, the average sizes of $z_{850^{-}}$and $Y_{105^{-}}$dropouts and the size of the $z \sim 12$ source, and for the fainter bin, the measured sizes of the stacks of $z_{850^{-}}$ and $Y_{105}$-dropouts with $L=(0.12-0.3) L_{z=3}^{*}$. In addition, we use the results reported in the literature: the average sizes at $z=2.5$ (Bouwens et al. 2004) and the average sizes at $z=4-6$ (Oesch et al. 2010a). ${ }^{17}$ We fit the following two functions to the data, $\log r_{e}=s \log (1+z)+a_{1}$ for $L=(0.3-1) L_{z=3}^{*}$ and $\log r_{e}=s \log (1+z)+a_{2}$ for $L=(0.12-0.3) L_{z=3}^{*}$, where $s$, $a_{1}$, and $a_{2}$ are free parameters. Varying the three parameters, we search for the best-fitting set of $\left(s, a_{1}, a_{2}\right)$ that minimizes $\chi^{2}$. The best-fit parameters are $s=-1.30_{-0.14}^{+0.12}, a_{1}=1.00_{-0.07}^{+0.09}$, and $a_{2}=0.88_{-0.09}^{+0.08}$. Note that the best-fit parameters do not change significantly even if the putative $z \simeq 12$ object is in the fainter UV luminosity bin, which might be the case when its $H_{160}$ magnitude is boosted by a strong line emission as Ellis et al. (2013) proposed (see also Brammer et al. 2013). We have checked that exclusion of the putative $z \simeq 12$ object produces no significant change in our results, but it is nevertheless interesting that its size conforms with the trend established at slightly lower redshifts. We note that these results are clearly consistent with the redshift trend derived by Oesch et al. (2010a) from the early UDF09 data, as they reported $s=-1.12 \pm 0.17$ for galaxies with luminosities in the range $L=(0.3-1) L_{z=3}^{*}$, and $s=-1.32 \pm 0.52$ for the fainter galaxies with $L=(0.12-0.3) L_{z=3}^{*}{ }^{18}$ However, our derived value for $s$ is more accurate, both because of the improved data and galaxy samples provided by UDF12, and because we have chosen to fit a single value of $s$ to both the bright and more modest luminosity galaxies.

It should be noted again that the above simple constant mass or constant velocity models assume that the stellar to halo size ratio does not change over this redshift range (Mo et al. 1998). To properly interpret our result, more realistic models are needed which carefully treat the stellar to halo size ratio, as well as consider effects on galaxy sizes from galaxy mergers, torquing, and feedback, based on a hierarchical galaxy formation scenario over the full redshift range (e.g., Somerville et al. 2008). These size measurements of high-redshift galaxies provide a launching point for a theoretical understanding of the structure of such galaxies, which has only recently been attempted but is of critical importance in understanding their properties (e.g., Muñoz \& Furlanetto 2012).

Figure 14 presents the average star formation surface density, $\Sigma_{\text {SFR }}$, as a function of redshift. Note that the measurements are shown up to $z \sim 8$ in Figure 14, because the uncertainty of the $z \sim 12$ measurements are too large to place a meaningful constraint. Our galaxies at $z>7$ have $\Sigma_{\mathrm{SFR}} \sim 2 . \Sigma_{\mathrm{SFR}}$ appears to increase toward high redshifts. In fact, this increase of $\Sigma_{\text {SFR }}$ is expected from the decreasing trend of galaxy size at a given luminosity (Figure 13). Since $\Sigma_{\mathrm{SFR}}$ is proportional to the UV luminosity density in the case of no dust extinction, Figure 14 indicates that UV luminosity surface brightness is higher for $z \sim 7-8$ galaxies than for $z \sim 4-5$ galaxies by a factor of $2-3$. Figure 14 has data points of dust-corrected $\Sigma_{\mathrm{SFR}}$. The dustextinction corrected $\Sigma_{\text {SFR }}$ is significantly larger than uncorrected $\Sigma_{\mathrm{SFR}}$ at $z \sim 4-5$, while almost no difference is found at $z>6$. Oesch et al. (2010a) claim that the dust-extinction corrected $\Sigma_{\mathrm{SFR}}$ is roughly constant over the redshift range of $z \sim 3-7$.

\footnotetext{
17 For the fitting, we do not utilize the GALFIT measurements by Oesch et al. (2010a), which they did not use as their fiducial ones. Note that the fitting result is consistent within $1 \sigma$, if we include the GALFIT measurements instead of their SExtractor measurements.

18 The results may indicate that the sizes of galaxies in the faint UV luminosity bin evolve faster than those in the bright UV luminosity bin, although the uncertainties in the fitting are large.
} 


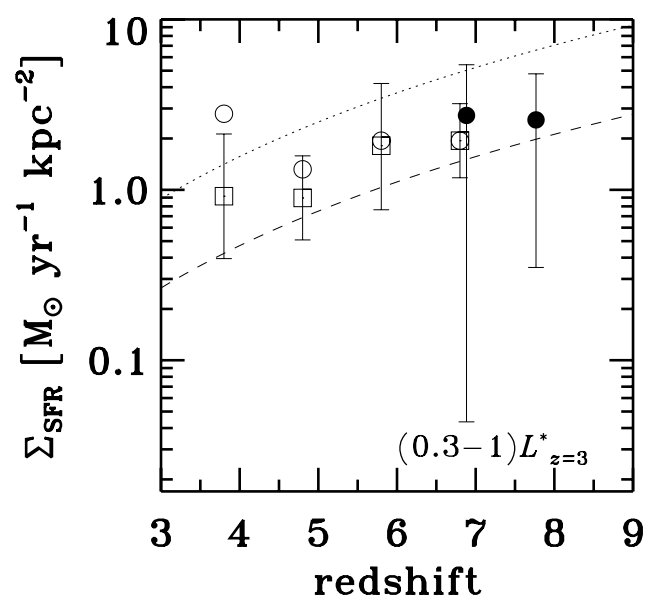

Figure 14. Evolution of the SFR surface density $\Sigma_{\mathrm{SFR}}$ as a function of redshift, for dropouts in the brightest luminosity bin, $L=(0.3-1) L_{z=3}^{*}$. The filled circles correspond to our $z_{850}$-dropouts and $Y_{105}$-dropouts. The open squares are taken from Oesch et al. (2010a), showing their dropout galaxies, while the open circles are the same objects but corrected for dust absorption. The dotted line and dashed line show the case with a constant UV luminosity $L=L_{z=3}^{*}$ and $0.3 L_{z=3}^{*}$, respectively, given that their half-light radii follow the simple relation of $(1+z)^{s}$, which is derived in Section 5 and shown in Figure 13.

Extending their study, we find this constant $\Sigma_{\mathrm{SFR}}$ up to $z \sim 8 .{ }^{19}$ Dotted and dashed lines in Figure 14 show the $\Sigma_{\text {SFR values for }}$ $L_{z=3}^{*}$ and $0.3 L_{z=3}^{*}$ expected from the best-fit size evolution of $(1+z)^{s}$. These lines imply that one would find galaxies with extremely high $\Sigma_{\mathrm{SFR}}$ at $z \gtrsim 10$ and beyond. A simple increase of the average $\Sigma_{\mathrm{SFR}}$ is not expected, however. This is because the typical UV luminosity, $L^{*}$, is fainter at higher redshifts and galaxies with $(0.3-1.0) L_{z=3}^{*}$ are quite rare at $z \gtrsim 10$. In this sense, even higher-redshift galaxies cannot take an extremely high average $\Sigma_{\mathrm{SFR}}$ beyond $\sim 3-10 M_{\odot} \mathrm{yr}^{-1} \mathrm{kpc}^{-2}$. In the local universe, the SFR surface densities of normal disk galaxies are about $0.01 M_{\odot} \mathrm{yr}^{-1} \mathrm{kpc}^{-2}$, smaller than what we have found for $z \sim 4-8$ dropouts. The centers of normal disk galaxies, on the other hand, reach about $1 M_{\odot} \mathrm{yr}^{-1} \mathrm{kpc}^{-2}$ (Figure 6 of Kennicutt 1998b; see also Momose et al. 2010), which is comparable to $\Sigma_{\mathrm{SFR}}$ of $z \sim 4-8$ dropouts. Note that some local starbursts show comparable $\Sigma_{\mathrm{SFR}}$ to $z \sim 4-8$ dropouts. However, because local starbursts, especially nuclear starbursts, have high $\Sigma_{\text {SFR }}$ up to $100-1000 M_{\odot} \mathrm{yr}^{-1} \mathrm{kpc}^{-2}$ (Kennicutt 1998b), the star formation surface density of dropout galaxies at $z \sim 4-8$ is significantly smaller than those of the extreme population found in the local universe, which indicates that star formation in dropout galaxies is not as rapid as that of local extreme starbursts. Speculatively, because high- $z$ dropouts are metaland dust-poor galaxies (e.g., Bouwens et al. 2012), gas cooling in a given amount of molecular clouds of high- $z$ dropouts would be less efficient than that of local starbursts.

\section{SUMMARY}

In this paper, we have presented sizes of dropout galaxy candidates at $z \sim 7-12$ identified by the 2012 Hubble Ultra Deep Field campaign. We have stacked the new F140W image with the existing F125W image and the deeper F160W image, to maximize the available depth at rest-frame wavelengths

\footnotetext{
19 Since the uncertainties are not insignificant, we cannot exclude the possibility that $\Sigma_{\mathrm{SFR}}$ is modestly decreasing with cosmic time. Ideally we seek improved statistical accuracy by increasing the number of $z \sim 4-8$ galaxies in the same luminosity range.
}

$\lambda_{\text {rest }} \simeq 1600-1700 \AA$ for $z_{850}$-dropout and $Y_{105}$-dropout samples respectively, allowing secure size measurement from $>15 \sigma$ detections. The extremely deep F105W data ensures that $z>8$ candidates are robust, extending the redshift range of reliable objects. We have performed surface brightness profile fitting for our samples at $z \sim 7-12$. Our measurements have shown that the average half-light radii of galaxies are very small, $0.3-0.4 \mathrm{kpc}$ at $z \sim 7-12$. Such sizes are, perhaps coincidentally, comparable to the sizes of GMAs in local star-forming galaxies.

Combining our new results at $z \simeq 7-12$ with existing average size measurements previously reported for dropout galaxies at $z \simeq 4-7$, we have investigated the size evolution of dropout galaxies. We have confirmed the trend for size to decrease with increasing redshift (at a given luminosity) and have shown that this trend appears to extend out to $z \simeq 12$. Motivated by the fact that, at least qualitatively, the sizes of the brighter $\left(0.3-1.0 L_{z=3}^{*}\right)$ and somewhat fainter $\left(0.12-0.3 L_{z=3}^{*}\right)$ dropout galaxies show a similar trend with redshift, we have attempted to model the size evolution of both samples together with a function of the form $(1+z)^{s}$ over the redshift range $z \simeq 4-12$. The result is a best-fitting parameter value of $s=-1.30_{-0.14}^{+0.12}$, approximately midway between the physically expected evolution for baryons embedded in dark halos of constant mass $(s=-1)$ and constant velocity $(s=-1.5)$. This evolution is consistent with that derived by Oesch et al. (2010a), albeit our derived evolution with redshift is slightly steeper than that derived by Oesch et al. (2010a) for the brighter galaxies. We have checked that our best-fitting value of $s$ is not significantly affected if the putative $z \simeq 12$ galaxy is excluded, but it is interesting that this object has a half-light radius which is perfectly consistent with our best-fitting relation.

We have also found that a clear size-luminosity relation, such as that found at lower redshift, is also evident in both our $z_{850^{-}}$and $Y_{105}$-dropout samples. This relation can be interpreted in terms of a constant surface density of star formation over a range in luminosity of $0.05-1.0 L_{z=3}^{*}$. These size-redshift and size-luminosity relations suggest that galaxy sizes at $z>4$ are not simply decided by the evolution of the constant mass or velocity of the parent halo and/or follow in the evolution of the stellar to halo size ratio with a similar SFR density.

Finally, our results also strengthen previous claims that the star formation surface density in dropout galaxies is broadly unchanged from $z \simeq 4$ to $z \simeq 8$ at $\Sigma_{\mathrm{SFR}} \simeq 2 \mathrm{M}_{\odot} \mathrm{yr}^{-1} \mathrm{kpc}^{-2}$. This value is $2-3$ orders of magnitude lower than that found in extreme starburst galaxies, but is very comparable to that seen today in the centers of normal disk galaxies. This provides further support for a steady smooth build-up of the stellar populations in galaxies in the young universe.

We thank the anonymous referee for constructive comments and suggestions. We also thank Rieko Momose and Suraphong Yuma for their helpful comments. This work was supported by Japan Society for the Promotion of Science (JSPS), Grants-in-Aid for Scientific Research (KAKENHI), Grant Numbers 24840010 and 23244025, and World Premier International Research Center Initiative (WPI Initiative), MEXT, Japan. E.F.C.L., A.B.R., and M.C. acknowledge the support of the UK Science and Technology Facilities Council. The U.S. authors acknowledge financial support from the Space Telescope Science Institute under award HST-GO-12498.01-A. J.S.D. and R.A.A.B. acknowledge the support of the European Research Council via the award of an Advanced Grant to J.S.D. J.S.D. also acknowledges the support of the Royal Society through a 
Wolfson Research Merit Award. S.C. acknowledges the support of the European Commission through the Marie Curie Initial Training Network ELIXIR. This work is based on data from the Hubble Space Telescope which is operated by NASA through the Space Telescope Science Institute via the association of Universities for Research in Astronomy, Inc., for NASA under contract NAS5-26555.

\section{REFERENCES}

Bertin, E., \& Arnouts, S. 1996, A\&AS, 117, 393

Bouwens, R. J., Illingworth, G. D., Blakeslee, J. P., Broadhurst, T. J., \& Franx, M. 2004, ApJL, 611, L1

Bouwens, R. J., Illingworth, G. D., Blakeslee, J. P., \& Franx, M. 2006, ApJ, 653,53

Bouwens, R. J., Illingworth, G. D., Franx, M., \& Ford, H. 2008, ApJ, 686,230

Bouwens, R. J., Illingworth, G. D., Labbe, I., et al. 2011a, Natur, 469, 504

Bouwens, R. J., Illingworth, G. D., Oesch, P. A., et al. 2010, ApJL, 709, L133

Bouwens, R. J., Illingworth, G. D., Oesch, P. A., et al. 2011b, ApJ, 737, 90

Bouwens, R. J., Illingworth, G. D., Oesch, P. A., et al. 2012, ApJ, 754, 83

Bouwens, R. J., Oesch, P. A., Illingworth, G. D., et al. 2013, ApJL, 765, L16

Brammer, G. B., van Dokkum, P. G., Illingworth, G. D., et al. 2013, ApJL, 765, L2

Bunker, A. J., Wilkins, S., Ellis, R. S., et al. 2010, MNRAS, 409, 855

Castellano, M., Fontana, A., Boutsia, K., et al. 2010, A\&A, 511, A20

de Jong, R. S., \& Lacey, C. 2000, ApJ, 545, 781

Dunlop, J. S., Rogers, A. B., McLure, R. J., et al. 2013, MNRAS, 432, 3520

Ellis, R. S., McLure, R. J., Dunlop, J. S., et al. 2013, ApJL, 763, L7

Ferguson, H. C., Dickinson, M., Giavalisco, M., et al. 2004, ApJL, 600, L107

Finkelstein, S. L., Papovich, C., Giavalisco, M., et al. 2010, ApJ, 719, 1250

Fontana, A., Vanzella, E., Pentericci, L., et al. 2010, ApJL, 725, L205

Grazian, A., Castellano, M., Fontana, A., et al. 2012, A\&A, 547, A51
Hathi, N. P., Malhotra, S., \& Rhoads, J. E. 2008, ApJ, 673, 686

Kennicutt, R. C., Jr. 1998a, ARA\&A, 36, 189

Kennicutt, R. C., Jr. 1998b, ApJ, 498, 541

Koekemoer, A. M., Ellis, R. S., McLure, R. J., et al. 2013, ApJS, 209, 3

Lorenzoni, S., Bunker, A. J., Wilkins, S. M., et al. 2011, MNRAS, 414, 1455

McLure, R. J., Dunlop, J. S., Bowler, R. A. A., et al. 2013, MNRAS, 432, 2696

McLure, R. J., Dunlop, J. S., Cirasuolo, M., et al. 2010, MNRAS, 403, 960

McLure, R. J., Dunlop, J. S., de Ravel, L., et al. 2011, MNRAS, 418, 2074

Mo, H. J., Mao, S., \& White, S. D. M. 1998, MNRAS, 295, 319

Mo, H. J., Mao, S., \& White, S. D. M. 1999, MNRAS, 304, 175

Mo, H. J., \& White, S. D. M. 2002, MNRAS, 336, 112

Momose, R., Okumura, S. K., Koda, J., \& Sawada, T. 2010, ApJ, 721, 383

Mosleh, M., Williams, R. J., Franx, M., et al. 2012, ApJL, 756, L12

Muñoz, J. A., \& Furlanetto, S. 2012, MNRAS, 426, 3477

Oesch, P. A., Bouwens, R. J., Carollo, C. M., et al. 2010a, ApJL, 709, L21

Oesch, P. A., Bouwens, R. J., Illingworth, G. D., et al. 2010b, ApJL, 709, L16

Oesch, P. A., Carollo, C. M., Stiavelli, M., et al. 2009, ApJ, 690, 1350

Oke, J. B., \& Gunn, J. E. 1983, ApJ, 266, 713

Ouchi, M., Mobasher, B., Shimasaku, K., et al. 2009, ApJ, 706, 1136

Peng, C. Y., Ho, L. C., Impey, C. D., \& Rix, H.-W. 2002, AJ, 124, 266

Peng, C. Y., Ho, L. C., Impey, C. D., \& Rix, H.-W. 2010, AJ, 139, 2097

Pirzkal, N., Sahu, K. C., Burgasser, A., et al. 2005, ApJ, 622, 319

Rand, R. J., \& Kulkarni, S. R. 1990, ApJL, 349, L43

Robertson, B. E., Furlanetto, S. R., Schneider, E., et al. 2013, ApJ, 768, 71

Schenker, M. A., Robertson, B. E., Ellis, R. S., et al. 2013, ApJ, 768, 196

Sersic, J. L. 1968, Atlas de Galaxias Australes (Cordoba: Observatorio Astronomico)

Somerville, R. S., Barden, M., Rix, H.-W., et al. 2008, ApJ, 672, 776

Steidel, C. C., Adelberger, K. L., Giavalisco, M., Dickinson, M., \& Pettini, M. 1999, ApJ, 519, 1

Tosaki, T., Shioya, Y., Kuno, N., et al. 2007, PASJ, 59, 33

Vogel, S. N., Kulkarni, S. R., \& Scoville, N. Z. 1988, Natur, 334, 402

Wilkins, S. M., Bunker, A. J., Lorenzoni, S., \& Caruana, J. 2011, MNRAS, 411, 23

Yan, H., Windhorst, R. A., Hathi, N. P., et al. 2010, RAA, 10, 867 